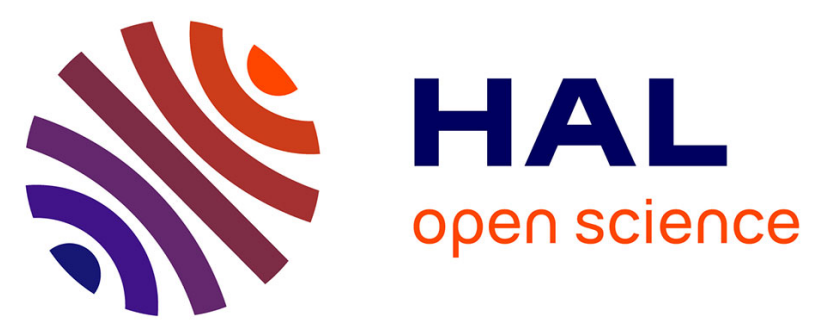

\title{
Large-scale computations of effective elastic properties of rubber with carbon black fillers
}

Aurélie Jean, François Willot, Sabine Cantournet, Samuel Forest, Dominique Jeulin

\section{- To cite this version:}

Aurélie Jean, François Willot, Sabine Cantournet, Samuel Forest, Dominique Jeulin. Large-scale computations of effective elastic properties of rubber with carbon black fillers. International Journal for Multiscale Computational Engineering, 2011, 9, pp.271-303. 10.1615/IntJMultCompEng.v9.i3.30 . hal-00661612

\section{HAL Id: hal-00661612}

https://hal-mines-paristech.archives-ouvertes.fr/hal-00661612

Submitted on 1 Dec 2021

HAL is a multi-disciplinary open access archive for the deposit and dissemination of scientific research documents, whether they are published or not. The documents may come from teaching and research institutions in France or abroad, or from public or private research centers.
L'archive ouverte pluridisciplinaire HAL, est destinée au dépôt et à la diffusion de documents scientifiques de niveau recherche, publiés ou non, émanant des établissements d'enseignement et de recherche français ou étrangers, des laboratoires publics ou privés.

\section{(c) (1) $\$$}

Distributed under a Creative Commons Attribution - NonCommerciall 4.0 International 


\title{
Large-scale computations of effective elastic properties of rubber with carbon black fillers
}

\author{
A. Jean, ${ }^{1,2}$, F. Willot, ${ }^{2}$ S. Cantournet, ${ }^{1}$ S. Forest, ${ }^{1}$ \& D. Jeulin ${ }^{2}$ \\ ${ }^{1}$ MINES ParisTech, Centre des Matériaux, CNRS UMR 7633, BP 8791003 Evry Cedex, France \\ ${ }^{2}$ MINES ParisTech, Centre de Morphologie Mathématique, Math'ematiques et Systèmes, 35 rue \\ St Honoré, F77305 Fontainebleau, France
}

\begin{abstract}
A general method, based on a multiscale approach, is proposed to derive the effective elastic shear modulus of a rub-ber with $14 \%$ carbon black fillers from finite element and fast Fourier transform methods. The complex multiscale microstructure of such material was generated numerically from a mathematical model of its morphology that was identified from statistical moments out of transmission electron microscopy images. For finite element computations, the simulated microstructures were meshed from three-dimensional reconstruction of the isosurface using the marching cubes algorithm with special attention to the quality of the topology and the geometry of the mesh. To compute the shear modulus and to determine the representative volume element, homogeneous boundary conditions were prescribed on meshes and combined with a domain decomposition method. Regarding parallel computing, specific difficulties related to the highly heterogeneous microstructures and complex geometry are pointed out. The experimental shear modulus (1.8 MPa) obtained from dynamic mechanical analysis was estimated by the Hashin-Shtrikman lower bound (1.4 MPa) and the computations on simulated microstructures $(2.4 \mathrm{MPa})$. The shear modulus was determined for two materi-als with the same volume fraction but different distribution of fillers. The current model of microstructures is capable of estimating the relative effect of the mixing time in processing associated with change in morphology on the elastic behavior. The computations also provide the local fields of stress/strain in the elastomeric matrix.
\end{abstract}

KEY WORDS: morphology, homogenization, meshing of microstructure, parallel computation, rubber elasticity, FEM, FFT, carbon black fillers

\section{INTRODUCTION}

The objective of the mechanics of microstructures is to derive the effective physical properties and the local fields of material volume element from the knowledge of the nature of the heterogeneities, their spatial distribution, and their constitutive laws (Cailletaud et al., 2003). The numerous methods for three-dimensional microstructure characterization provide detailed information concerning the three-dimensional morphology of the microstructure or the nature of their components (Ludwig et al., 2009). Detailed information about the morphology can be introduced in a finite element (FE) model to compute the mechanical properties of the microstructure. In order to describe with precision the geometry of the microstructure, the resulting FE mesh contains a large number of nodes and considerable numerical effort is necessary to solve the corresponding value problem, for instance, based on parallel computing.

The homogenization methods are used to derive the effective physical properties from information on the microstructure at the microscopic scale. For two-phase heterogeneous materials and for linear elasticity behavior, two groups of homogenization techniques are usually considered: the analytical techniques and the numerical ones. In the analytical techniques family, it is possible to bound the effective property (Beran, 1968) using first-order Voigt and Reuss bounds, second-order bounds (Hashin and Shtrikman, 1963), or higher order bounds usually containing more 
information on the morphology of the microstructure (Jeulin and Ostoja-Starzewski, 2001). More and more statistical information about the microstructure morphology is also considered and leads to a hierarchy of bounds (Kröner, 1980; Torquato and Stell, 1983; Torquato and Lado, 1986; Torquato, 1991). It is also possible to estimate directly the effective properties with the Mori-Tanaka model, for instance, Mori and Tanaka (1973), or using a self-consistent scheme refering to disordered materials, for electrical conductivity Bruggeman (1935) and Beran (1968), and for linear behavior (Kröner, 1958; 1977). More recently, a self-consistent scheme has been developed in order to estimate the properties of a rubber with carbon black (CB) fillers at large deformations (Omnes et al., 2008a). Regarding the numerical techniques, the integrated methods and sequential methods are proposed. In the case of the integrated method, the multiscale character is directly introduced in the FE simulation, leading to two levels (micro and macro) of computations, such as the $\mathrm{FE}^{2}$ method (Feuel and Chaboche, 2000) or the Arlequin technique (Ben Dhia, 1998). In the case of the sequential methods, the homogenization problem is solved using simulations on samples of microstructures for which the representative volume element (RVE) plays a key role. The RVE is defined as the volume of heterogeneous material that is large enough to be statistically representative of the material. Sab (1992) demonstrated that the physical responses obtained for different homogeneous boundary conditions prescribed on the sample coincide for any volume larger than the RVE. For a given physical property, the size of the RVE can be determined from a statistical and numerical approach (Kanit et al., 2003) using a Monte Carlo procedure to compute the apparent response on realizations of simulations of microstructures for increased sizes.

For the determination of the RVE of a heterogeneous material, a model of the morphology (Serra, 1982) is determined based on two- or three-dimensional (2D/3D) information on the geometry of the microstructure [for instance, microtomography data (Madi et al., 2007; Ludwig et al., 2009); or scanning electron or transmission electron microscopy or (SEM/TEM) images]. Many papers referred to mathematical models of the morphology of heterogeneous materials to derive properties by homogenization such as Voronoi tessellation for polycrystals (Barbe et al., 2001) and granular media (Sab and Boumediene, 2005), or hard core sphere models to represent aggregates in cement paste (Wriggers and Moftah, 2006; Hain and Wriggers, 2008). For rubber, the CB aggregates were modeled as squares or dodecaedron in a elastomeric matrix in Bergstrom and Boyce (1998) and as the union of CB spherical-shaped particles in Naito et al. (2007). In Jha et al. (2007) a cuberille model was considered to describe the aggregates surrounded by a third phase representing the bonded rubber. In Laiariandrasana et al. (2009) a composite model was also obtained from periodic homogenization by placing a spherical CB particle at the center of a tetrakaidecaedron cell. In general, all these works have shown the need for a three-dimensional microstructural model to take the interaction between aggregates and the interaction of fillers with the elastomeric matrix into account.

The goal of the present work is to use a 3D morphological model and estimate the effective elastic shear modulus of a rubber with CB fillers based on large-scale FE computations. The addition of filler aggregates (e.g., carbon black or silica) to rubber leads to significant improvements of the physical and the mechanical properties of the material. These mechanisms of elastomer reinforcement by fillers also contribute to an increase of strain-induced mechanical nonlinearities. For example, if a dynamic load is applied at medium-to-large strains, the filler network can deteriorate, resulting in a loss in stiffness. This well-known behavior is called the Payne effect (Payne and Whittaker, 1971). Some experiment data obtained from a dynamic mechanical analyzer (DMA) (Jean, 2009) show that the Payne effect is enhanced when the initial shear modulus increases. The shear modulus increases when the volume fraction of fillers increases and tends to decrease when the time of mixing of fillers into the matrix increases for a constant volume fraction. Much effort has been devoted to reducing the Payne effect without compromising the enhancement of the other important material properties. Another related effect is commonly observed in filled elastomers at large strains, namely, stress softening. This phenomenon, known as the Mullins effect (Mullins, 1947; Mullins and Tobin, 1954), is usually assumed to be either the result of an evolution in the hard and soft domain of the microstructure, whereby the effective volume fraction of the soft domain increases with stretch, or the result of irreversible damage in the material, or a combination of both phenomena. But the basic mechanisms of elastomer reinforcement by fillers, including the way it is affected by the particle size or the degree of dispersion, are not very well documented. Since this paper is focused on the prediction of the effective elastic shear modulus at small deformation, the destruction of the carbon-black network is not taken into account. For further insight into experimental results and modelling approaches of Mullins and Payne effects, we recommend the following references: Govindjee and Simo, 2009; Ogden and Roxburgh, 1999; Diani et al., 2009; Cantournet et al., 2009; Medalia, 1978; Kraus, 1984; Lion, 1996. 
Section 2 presents the morphological model of the microstructure, identified on the statistical moments on TEM images, and depicts the method retained to generate a three-dimensional mesh of the microstructure. Section 3 defines the general boundary value problem for the determination of effective linear properties of the simulated meshed microstructures. Section 4 proposes a strategy of computation using the domain decomposition method and parallel computing applied to the highly heterogeneous rubber composite. The results of the determination of the shear modulus and of the size of the following RVE are given in Section 5. In addition to the FE computations, the elastic response and RVE from periodic computations were obtained using the fast fourier transform (FFT) approach (Michel et al., 2000). Finally, the computations also give insight into the local stress and strain fields in the elastomeric matrix between CB aggregates as shown in Section 5.3.

\section{REPRESENTATION OF THE MICROSTRUCTURE OF RUBBER WITH CB FILLERS}

\subsection{Multi-Scale and Statistical Modeling of the Microstructure}

\subsubsection{Morphological Modeling Approach}

To model the structure of a rubber with carbon black fillers, two kinds of models can be investigated. The first kind consists of describing the microstructure as an ideal periodic composite using periodic computations on an elementary cell. This method, widely used in the case of two dimensions (Zeman and Sejnoha, 2001), was also extended in three dimensions by Jean (2009) and Laiarinandrasana et al. (2009), where a carbon black particle was located at the center of a tetrakaidecaedron cell in order to derive the effective behavior of a centered cubic symmetry of fillers in a rubber matrix. Hereby, such models do not take the aggregates of carbon black fillers into account. The second kind of model consists of simulating more realistic three-dimensional microstructures, strongly motivated by the large data on the three-dimensional morphology and the increase of capabilities of computations. A first set of models consists of tessellating space into cells. For instance, the Voronoi cell tesselation is used in the case of polycrystals (Barbe et al., 2001; Osipov et al., 2008; Gérard et al., 2009) and granular media (Sab and Boumediene, 2005). A second set of models consists of describing the dispersion of inclusions and/or pores in a matrix such as in Hain and Wriggers (2008), where a model of hard-core spheres represented the aggregates in a cement paste. In the present study, the second set of models was retained to describe the distribution of aggregate fillers in the rubber matrix.

\subsubsection{Multiscale Boolean-Cox Model}

Figure 1(a) shows a TEM micrograph of $1500 \mathrm{~nm}$ in length of the microstructure of a rubber containing $14 \%$ fillers. This image reveals clearly the multiscale microstructure of this material with three scales: the spherical-shaped parti-

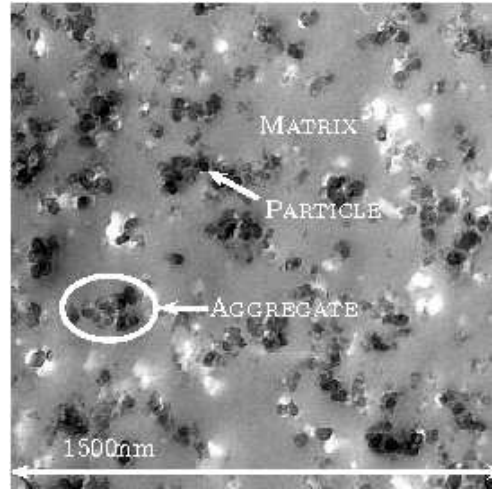

(a)

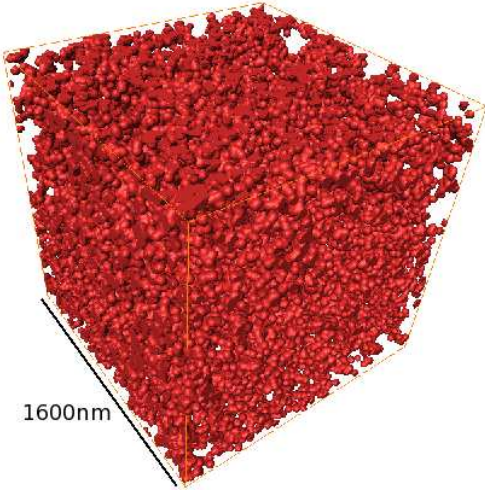

(b)

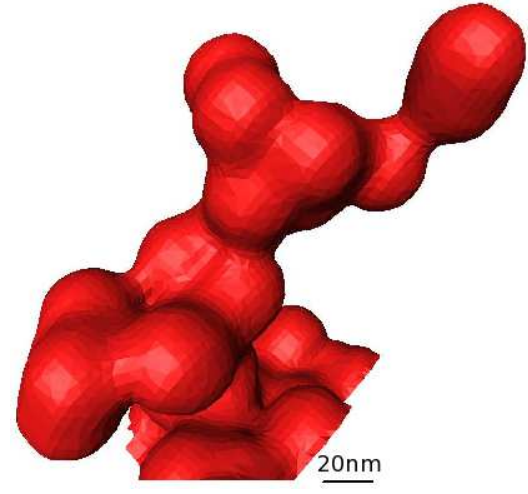

(c)

FIG. 1: Statistical modeling of rubber with carbon black fillers: (a) electron micrograph, (b) simulation of microstructure of $1600 \mathrm{~nm}$ in length, and (c) focus on a single aggregate. 
cle, the aggregate, and the matrix located between aggregates. A multiscale model was favored to represent each scale of the microstructure (Jeulin, 1991; Jeulin and Le Coënt, 1995; Savary et al., 1999; Delarue, 2001). For the present material, the multiscale morphological model was detailed in (Jean et al., 2007; 2010) and consisted of three Boolean models. A Boolean model of spheres, associated with a truncated Gaussian distribution of the radius, describes the carbon black particles. According to experimental measurements of the morphology of aggregates, a Boolean model of ellipsoids, associated with a log-normal distribution of their size, was retained to describe the aggregates. Finally, a Boolean model of spheres with a constant radius represents the matrix zones. The final microstructure was obtained by a combination of Boolean models according to a Cox algorithm (Moreaud and Jeulin, 2005; Jean et al., 2007). Figure 1(b) illustrates a simulation of a microstructure of the CB aggregates of the rubber composite of $1600 \mathrm{~nm}$ length, the elastomeric matrix corresponding to the complementary set. Figure 1(c) shows an example of aggregate and its own structure. This model contains four parameters to identify: the volume fraction of each scale (particles, aggregates and matrix) and the radius of the spheres describing the matrix zones.

\subsubsection{Identification of the Boolean Cox Model from TEM Images}

To identify the parameters of the multiscale model, an original method was proposed in Jean et al. $(2007 ; 2010)$. This method involves two steps. The first step aims at fitting a first primary set of parameters on an approximate expression of the analytic covariance. The obtained set of parameters is then used in the second step as an initial set. In this second step, the model is identified by minimizing the error on the statistical moments (covariance and third-order moment) and the closing curve, providing a size distribution of the matrix. This optimization consists of computing a representative sample of virtual TEM images from 3D simulations of microstructure and comparing the numerical morphological data to the experimental data. Finally, the identified model is validated according to some macroscopic percolation criterion.

\subsection{FE Meshing of Microstructures}

\subsubsection{General Overview}

Several techniques are available to mesh a structure or a microstructure within the FE method. In the case of heterogeneous media with complex morphology, two methods are considered here. In the first method, the isosurface (the surface between two phases) is explicitly discretized so that the element edges of the mesh follow the interface between the two phases. In the second method, the isosurface is not discretized but the mesh properties are modified in the areas where the isosurface should be located. One possible choice consists of assigning different material properties to each integration point in a single element crossed by the isosurface (Lippman et al., 1997; Barbe et al., 2001). This is the so-called multiphase elements technique. Other recent techniques introduce a discontinuity in the FE interpolation of the corresponding elements. This enrichment is local in the embedded FE method (Ortiz et al., 1987) or global as in the extended finite element method (X-FEM) (Moës et al., 1999), where the isosurface is located using the level sets method. This method, applied to the hard core model of spheres, gives promising results (Ionescu et al., 2007). However, its robustness has not yet been demonstrated on large-scale simulations of heterogeneous microstructures.

Hence in the present work, priority has been given to generate an FE mesh that follows the microstructure interfaces. This is called the conforming meshing technique (Frey and Georges, 1999). Here, the geometry of simulated microstructures was given by a list of the radii and coordinates of the center of all carbon black spherical particles. In similar cases, where an analytic expression of the isosurface is available, computer-aided design (CAD) methods are favored to create the geometrical objects describing the isosurface. These geometrical objects are then meshed using Delaunay triangulations (Georges and Borouchaki, 1997). The same techniques or the advanced front method (Lohner, 1996) allow the mesh to eventually be filled in volume. In the present case, the union operation of spheres, according to the Boolean model, was not robust enough when a few dozens of intersected particles were considered. Therefore, a concurrent method to CAD techniques was retained the marching-cubes algorithm (Lorensen and Cline, 1987). This algorithm reconstructs the isosurface from the three-dimensional image. 


\subsubsection{Triangulation of Interface and Tetrahedral Meshing}

The three-dimensional image of a simulated microstructure of rubber composite was composed by a set of twodimensional images of one-pixel thickness [Fig. 2(a)]. These images were binarized, the white and black labels corresponding to the carbon particles and the rubber matrix, respectively, leading to a voxel-based discretization of the particles [Fig. 2(b)] and of the matrix [Fig. 3(a)]. The isosurface describing the interface between the two materials was captured using the marching cubes algorithm. The quality of the isosurface reconstruction is strongly related to the resolution of the image. In this algorithm, a cube of the size of one voxel is translated along the three vectors $(\underline{x}, \underline{y}, \underline{z})$ so that each vertex matches with the center of a voxel. At each step, the label of the eight vertices is tested. When the labels differ, the isosurface crosses the cube; thus, an elementary polygonal surface, chosen in a table containing all the possible configurations, is built up in the cube. Finally, the union of all the elementary polygons creates the final three-dimensional polygonal isosurface. One should notice that the marching cubes procedure considers the outside volume of the microstructure as a third phase. The outer boundary of the volume of simulation is thus included

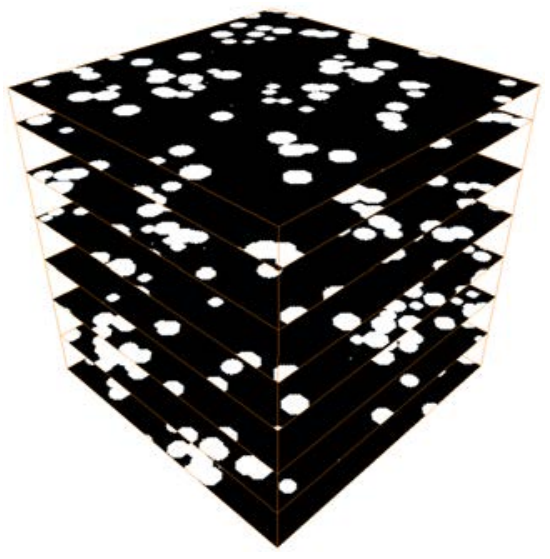

(a)

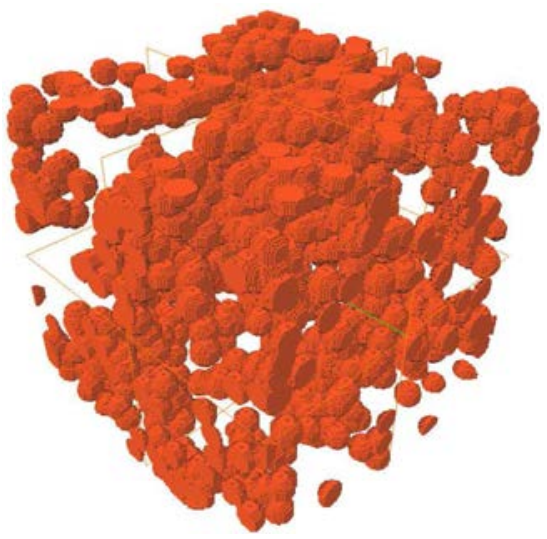

(b)

FIG. 2: (a) Set of two-dimensional images constituting the three-dimensional simulation and (b) discretization into voxels of three-dimensional image simulation (carbon black fillers).

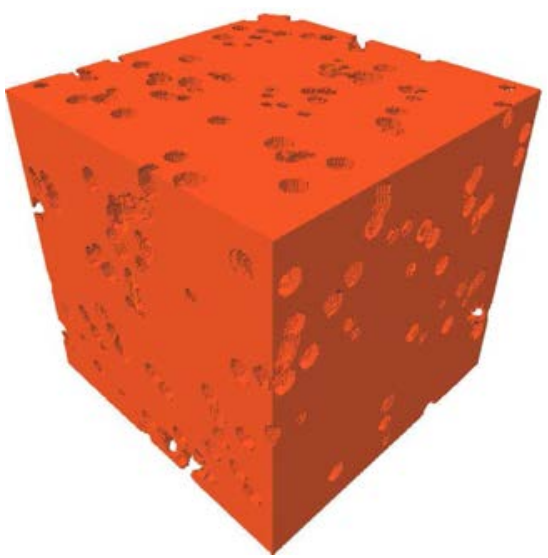

(a)

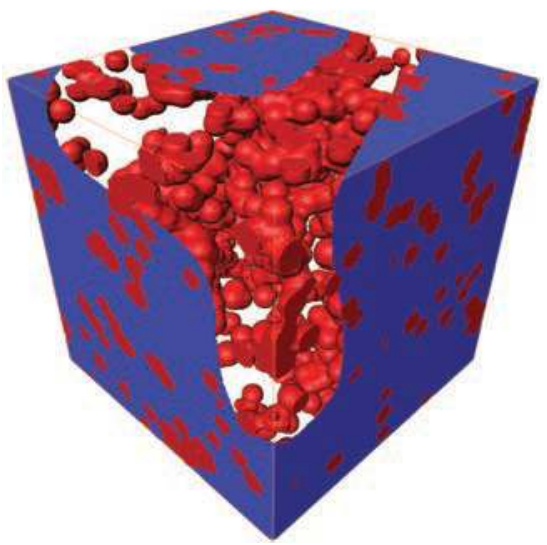

(b)

FIG. 3: (a) Discretization into voxels of three-dimensional image simulation (elastomeric matrix) and (b) representation of three-dimensional polygonal surface using the marching cubes algorithm. 
in the final polygonal surface [Fig. 3(b)]. The whole procedure was carried out with Avizo software (Vizualization Science Group, 2009).

The obtained polygonal surface [Fig. 4(a)] was then discretized by triangular-shaped elements. The corresponding mesh [Fig. 4(b)] usually contains a large number of nodes. For instance, for a simulated microstructure of $1.25 \times 10^{5}$ voxels with about 20 spheres, the mesh of the isosurface contained around $10^{6}$ elements [Fig. 5(a)]. Such a fine discretization may be required for a precise description of the particles. The faces of the cube also belong to the isosurface. There is no need for a fine mesh on these faces where only matrix material is present. This first mesh also contains too many flat triangles with acute angles on the surface of particles, as shown in Fig. 4(b), contrary to the outer boundary of the volume [Fig. 5(a)]. Also, on the union area between intersected particles, some overlaping elements were detected by Avizo. Because of its size and its bad quality, this surface mesh cannot be used for filling with volumic elements and for FE computations. It was proposed first to coarsen the surface mesh uniformly on the faces of the cube, preserving fine enough discretization at the interface between the matrix and particles [Fig. 5(b)]. Then, in order to have a surface mesh with a correct topology, a manual correction of flat-shaped elements and intersected elements had to be carried out.

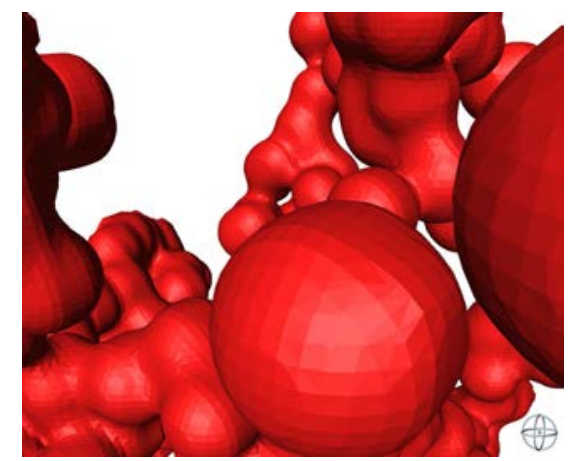

(a)

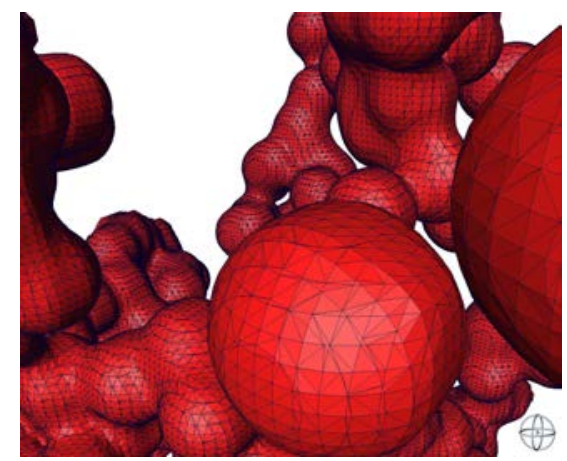

(b)

FIG. 4: (a) Focus on a sphere from three-dimensional microstructure and (b) its corresponding first primary FE mesh obtained from marching cubes algorithm.

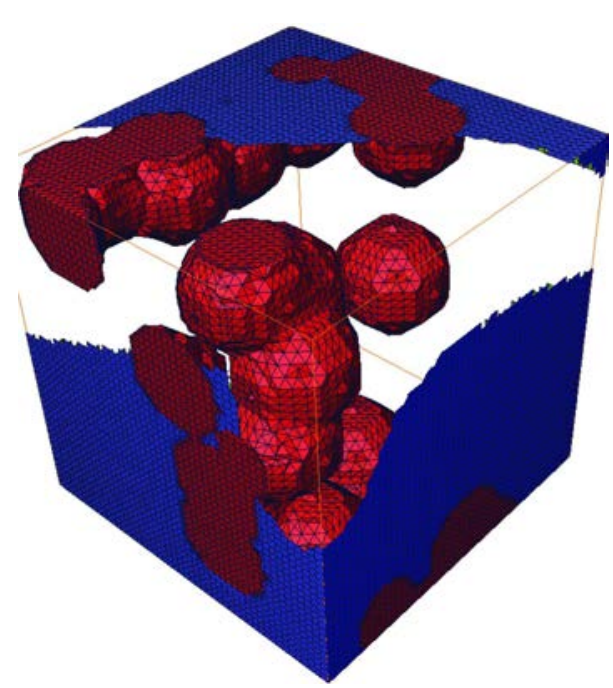

(a)

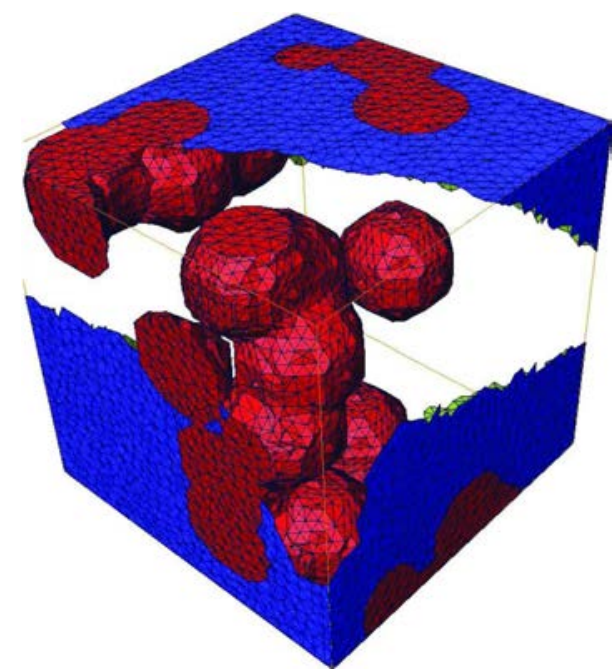

(b)

FIG. 5: Mesh of microstructure before (a) and after (b) reduction of the mesh size. 
Next, this surface mesh could be filled to obtain a three-dimensional mesh for the FE computation. Nevertheless, for practical reasons regarding domain decomposition methods (see Section 4.3), this surface mesh has to be further optimized. This step was automatically conduced by the Yams software (INRIA, 2009) interfaced with a Zset environment (Mines-ParisTech, 2003). Two tasks were considered in the procedure. The first task aims at refining the surface mesh at the interface between the two materials and coarsening it inside the phases (Fig. 6). Attention was paid to the smoothness of the element size gradient from fine to coarse mesh domains. The second task deals with the optimization of the classic geometrical criteria (Frey and Georges, 1999) defined for each element. The elements that do not satisfy the criteria were modified by moving their nodes and/or changing their connectivity. Finally, a free linear tetrahedral mesh was built on the basis of the conforming optimized surface triangulation. The meshing was performed using Delaunay triangulation based on the TetMesh GHS3D module (INRIA, 2009). Figures 7(a) and 7(b) illustrate

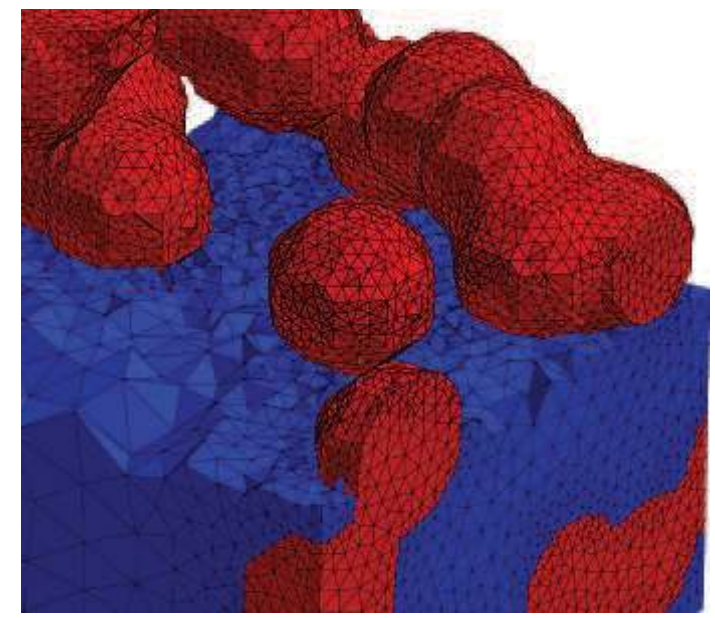

FIG. 6: Three-dimensional FE meshing of computed 3D microstructures, focusing on geometrical progression of meshsize in the matrix (blue phase).

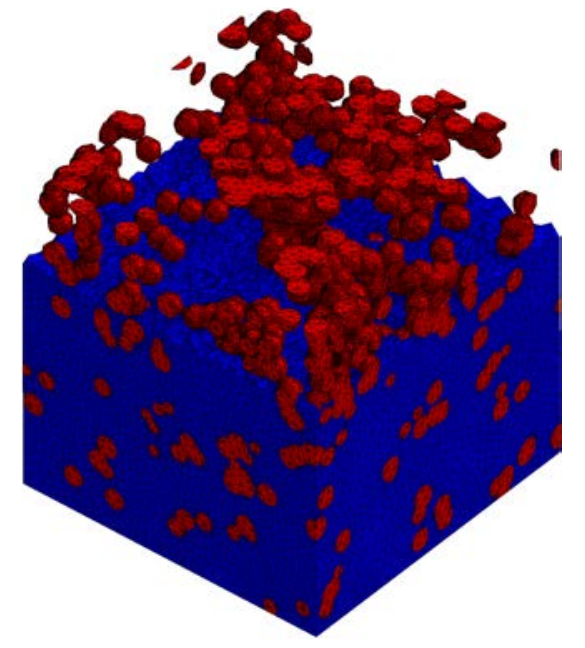

(a)

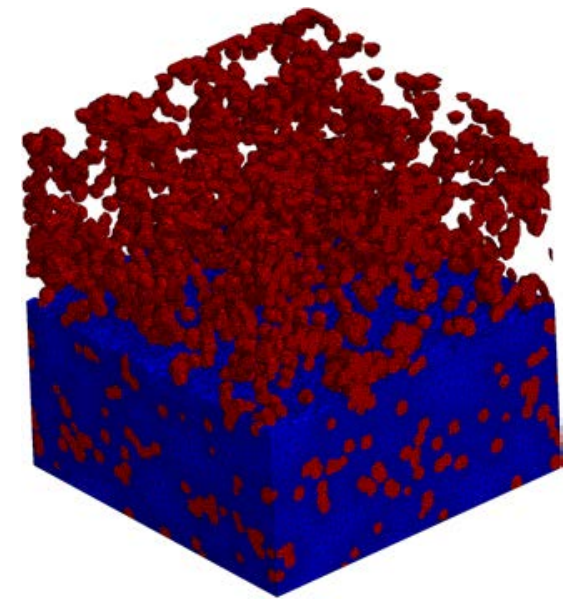

(b)

FIG. 7: Three-dimensional FE meshing of computed 3D microstructures - (a) simulation of $600 \mathrm{~nm}$ in length, 2241 particles and 1,046, 040 nodes: (b) simulation of $1000 \mathrm{~nm}$ in length, 4061 particles, and 1, 412, 997 nodes. 
such three-dimensional FE meshes for a simulated microstructure of $600 \mathrm{~nm}$ and $1000 \mathrm{~nm}$ length, respectively. Both contain more than $10^{6}$ nodes.

\section{BOUNDARY VALUE PROBLEM FOR THE DETERMINATION OF EFFECTIVE LINEAR PROPERTIES}

\subsection{Apparent vs Effective Properties}

A volume $V$ is considered made of heterogeneous material. Its outer boundary is $\partial V$. A local behavior is defined at every point $\underline{\boldsymbol{x}}$ belonging to the volume $V$, with $\underset{\sim}{\boldsymbol{c}}(\underline{\boldsymbol{x}})$ the local fourth-rank tensor of elasticity moduli:

$$
\sigma(\underline{\boldsymbol{x}})=\underset{\sim}{\boldsymbol{c}}(\underline{\boldsymbol{x}}): \underset{\sim}{\boldsymbol{\varepsilon}}(\underline{\boldsymbol{x}}) \quad \forall \underline{\boldsymbol{x}} \in V
$$

The apparent mechanical response is calculated after averaging the strain and stress tensors over the volume $V$ :

$$
\underset{\sim}{\boldsymbol{\Sigma}}=\frac{1}{V} \int_{V} \underset{\sim}{\boldsymbol{\sigma}}(\underline{\boldsymbol{x}}) d V \quad \underset{\sim}{\boldsymbol{E}}=\frac{1}{V} \int_{V} \underset{\sim}{\stackrel{\varepsilon}{\sim}}(\underline{\boldsymbol{x}}) d V
$$

Due to the linearity of the elasticity problem, the fourth-rank tensor of apparent elasticity moduli ${\underset{\sim}{a p p}}^{a \text { can be defined }}$ as

$$
\underset{\sim}{\boldsymbol{\Sigma}}=\underset{\sim}{\boldsymbol{\sim}}{ }^{a p p}: \underset{\sim}{\boldsymbol{E}}
$$

The Voigt notation for the elasticity tensor is

$$
\left(\begin{array}{c}
\Sigma_{11} \\
\Sigma_{22} \\
\Sigma_{33} \\
\Sigma_{12} \\
\Sigma_{23} \\
\Sigma_{31}
\end{array}\right)=\left[\begin{array}{cccccc}
C_{11}^{a p p} & C_{12}^{a p p} & C_{13}^{a p p} & C_{14}^{a p p} & C_{15}^{a p p} & C_{16}^{a p p} \\
- & C_{22}^{a p p} & C_{23}^{a p p} & C_{24}^{a p p} & C_{25}^{a p p} & C_{26}^{a p p} \\
- & - & C_{33}^{a p p} & C_{34}^{a p p} & C_{35}^{a p p} & C_{36}^{a p p} \\
- & - & - & C_{44}^{a p p} & C_{45}^{a p p} & C_{46}^{a p p} \\
- & - & - & - & C_{55}^{a p p} & C_{56}^{a p p} \\
- & - & - & - & - & C_{66}^{a p p}
\end{array}\right]\left(\begin{array}{c}
E_{11} \\
E_{22} \\
E_{33} \\
2 E_{12} \\
2 E_{23} \\
2 E_{31}
\end{array}\right)
$$

When the volume $V$ is an RVE, the apparent response coincides with the effective mechanical behavior of the heterogeneous material. In the present work, three types of boundary conditions to be prescribed on the boundary $\partial V$ are considered.

\subsection{Boundary Conditions}

\subsubsection{Kinematic Uniform Boundary Conditions}

The displacement $\underline{\boldsymbol{u}}$ is prescribed at each point $\underline{\boldsymbol{x}}$ belonging to the boundary $\partial V$ such that

$$
\underline{\boldsymbol{u}}=\underset{\sim}{\boldsymbol{E}} \underline{\boldsymbol{x}} \quad \forall \underline{\boldsymbol{x}} \in \partial V
$$

where $\underset{\sim}{\boldsymbol{E}}$ is a given homogeneous strain tensor. It follows that

$$
\langle\underset{\sim}{\varepsilon}\rangle=\frac{1}{V} \int_{V} \underset{\sim}{\varepsilon} d V=\underset{\sim}{\boldsymbol{E}}
$$

The macroscopic stress tensor is then defined by the spatial average:

$$
\langle\underset{\sim}{\boldsymbol{\sigma}}\rangle=\frac{1}{V} \int_{V} \underset{\sim}{\boldsymbol{\sigma}} d V=\underset{\sim}{\boldsymbol{\Sigma}}
$$




\subsubsection{Static Uniform Boundary Conditions}

The traction vector $\underline{\sigma} \cdot \underline{\boldsymbol{n}}$ is prescribed at the boundary $\partial V$ such that

$$
\underline{\sigma} \cdot \underline{\boldsymbol{n}}=\underset{\sim}{\boldsymbol{\Sigma}} . \underline{\boldsymbol{n}} \quad \forall \underline{\boldsymbol{x}} \in \partial V
$$

where $\Sigma$ is a given homogeneous stress tensor. It follows that

$$
\langle\boldsymbol{\sigma}\rangle=\frac{1}{V} \int_{V} \underset{\sim}{\boldsymbol{\sigma}} d V=\underset{\sim}{\boldsymbol{\Sigma}}
$$

The macroscopic strain tensor is then defined by the spatial average:

$$
\langle\underline{\sim}\rangle=\frac{1}{V} \int_{V} \underset{\sim}{\varepsilon} d V=\underset{\sim}{\boldsymbol{E}}
$$

\subsubsection{Periodic Conditions}

The displacement field over the entire volume $V$ takes the following form considering a periodic fluctuation $\underline{\boldsymbol{v}}$ (Kanit et al., 2003):

$$
\underline{\boldsymbol{u}}=\underset{\sim}{\boldsymbol{E}} \cdot \underline{\boldsymbol{x}}+\underline{\boldsymbol{v}}
$$

The fluctuation $\underline{\boldsymbol{v}}$ is periodic in the sense that it takes the same value at two homologous points at opposite faces of $\partial V$. Furthermore, the volume $V$ is in equilibrium because the traction vector $\underline{\sigma} \cdot \underline{\boldsymbol{n}}$ takes opposite values at two homologous points at opposite faces of $\partial V$.

The periodicity conditions were not used in the FE simulations reported in this work because of the difficulty of constructing large meshes with homologous nodes at the boundary. On the other hand, the use of Lagrange multipliers to enforce the periodicity conditions turns out to be too expensive for the considered parallel computations. Nevertheless, an alternative method allows computations of the apparent response of a volume with periodic conditions (PC), based on the FFT method (Moulinec and Suquet, 1994; Willot and Jeulin, 2009), which consists of computing the local fields from an image of the periodic simulation (Section 3.6).

\subsection{Bounds of Effective Moduli by Apparent Properties}

One recalls that the effective behavior is defined for a representative volume element. It is possible to bound the effective moduli by the apparent responses obtained for smaller volumes (Huet, 1990). Let $\underset{\sim}{\boldsymbol{c}}(\underline{\boldsymbol{x}})$ and $\underset{\sim}{\boldsymbol{s}}(\underline{\boldsymbol{x}})$ be the fourth-rank tensor fields of elastic moduli and compliances in the volume $V$ of heterogeneous material, defined by Eq. (1).

For the kinematic uniform boundary condition (KUBC), problem

$$
\underset{\sim}{\boldsymbol{\Sigma}}=\langle\underset{\sim}{\boldsymbol{\sigma}}\rangle=\langle\underset{\sim}{\boldsymbol{c}}: \underset{\sim}{\boldsymbol{A}}: \underset{\sim}{\boldsymbol{E}}\rangle=\langle\underset{\sim}{\boldsymbol{c}}: \underset{\sim}{\boldsymbol{A}}\rangle: \underset{\sim}{\boldsymbol{E}}=\underset{\sim}{\boldsymbol{C}_{E}^{a p p}}: \underset{\sim}{\boldsymbol{E}}
$$

For the static uniform boundary condition (SUBC) problem,

$$
\underset{\sim}{\boldsymbol{E}}=\langle\underset{\sim}{\mathcal{\varepsilon}}\rangle=\langle\underset{\sim}{\boldsymbol{s}}: \underset{\sim}{\boldsymbol{\sim}}: \underset{\sim}{\boldsymbol{\Sigma}}\rangle=\langle\underset{\sim}{\boldsymbol{s}}: \underset{\sim}{\boldsymbol{B}}\rangle: \underset{\sim}{\boldsymbol{\Sigma}}=\underset{\sim}{\boldsymbol{S}} \boldsymbol{\sim}^{a p p}: \underset{\sim}{\boldsymbol{\Sigma}}
$$

$\underset{\sim}{\boldsymbol{A}}$ and $\underset{\sim}{\boldsymbol{B}}$ are the fourth-rank concentration tensors such that

$$
\left\{\begin{array}{l}
\underset{\sim}{\boldsymbol{\varepsilon}}(\underline{\boldsymbol{x}})=\underset{\sim}{\boldsymbol{A}}(\underline{\boldsymbol{x}}): \underset{\sim}{\boldsymbol{E}} \\
\underset{\sim}{\boldsymbol{\sigma}}(\underline{\boldsymbol{x}})=\underset{\sim}{\boldsymbol{B}}(\underline{\boldsymbol{x}}): \underset{\sim}{\boldsymbol{\Sigma}}
\end{array}\right.
$$

From Eq. (2), the concentration tensors satisfy

$$
\langle\underset{\sim}{\boldsymbol{A}}\rangle=\langle\underset{\sim}{\boldsymbol{B}}\rangle=\underset{\approx}{\boldsymbol{I}}
$$

where $\underset{\approx}{\boldsymbol{I}}$ is the fourth-rank identity tensor. 
The fourth-rank tensors $\underset{\sim}{\boldsymbol{\sim}}{ }_{E}^{a p p}$ and $\underset{\sim}{\boldsymbol{S}}{ }_{\Sigma}^{a p p}$ define the apparent moduli computed with KUBC and apparent compliance computed with SUBC, respectively, for a given volume $V$. For volumes smaller than the representative volume element, the effective fourth-rank tensor of elasticity moduli is bounded as follows (Huet, 1990):

$$
\underset{\approx}{\operatorname{S}}{ }_{\Sigma}^{a p p-1} \leq{\underset{\sim}{\boldsymbol{C}}}^{e f f} \leq \underset{\approx}{\boldsymbol{C}}{ }^{a p p}
$$

where ${\underset{\sim}{\boldsymbol{i}}}^{a p p}$ and $\underset{\sim}{\boldsymbol{S}}{ }^{a p p}$ are the tensors of apparent elastic moduli and compliances, respectively. This inequality is written in the sense of quadratic forms. For large enough volumes, i.e., for representative volumes, the properties obtained for every boundary condition are equivalent (Sab, 1992):

$$
\underset{\approx}{\boldsymbol{C}}{ }^{a p p}=\underset{\approx}{\boldsymbol{S}}{ }_{\Sigma}^{a p p-1}={\underset{\approx}{\boldsymbol{C}}}^{e f f}={\underset{\sim}{\boldsymbol{S}}}^{e f f-1}
$$

This condition is used in (Ranganathan and Ostoja-Starzewski, 2008) to detect the size of the RVE. It has to be completed by the statistical precision of the effective properties resulting from the fluctuations of microstructure, as made in Kanit et al. (2003), Jeulin (2005), and Jeulin and Moreaud (2008). Since we estimated only $C_{44}, C_{55}$, and $C_{66}$ for simplification of the calculations, we could not check the fact that the KUBC and SUBC tensors are inverse of each other, as proposed in Ranganathan and Ostoja-Starzewki (2008). In the case of the simulated microstructures of rubber with carbon black fillers, the size of the (RVE) for the shear modulus is not known a priori; hence the shear moduli obtained for each boundary condition has to be compared. Usually the difference between the responses of the dual boundary conditions tends to increase when the size of the simulated microstructure decreases. In Section 4.2 we make use of the statistical approach of the RVE on our simulations.

\subsection{Elastic Properties of Constituents}

The elastic properties of both phases, carbon black fillers and elastomeric matrix, were given by Michelin. For the rubber, the Young's modulus was determined by Michelin from dynamic mechanical analysis testing and the Poisson ratio was given by Michelin. These results $\left(E_{M}=3 \mathrm{MPa}\right.$ and $\left.v_{M}=0.49983\right)$ are in agreement with the values found in literature (Holownia, 1974; Omnes et al., 2008b). The rubber exhibits a quasi-incompressible behavior. Concerning the carbon black fillers, the Poisson ratio is usually taken as $v_{C B}=0.3$. Due to the difficulty in carring out mechanical tests on the fillers alone, the Young's modulus of the carbon black fillers is chosen as that of the carbon black and does not take the specific structure of the carbon black particle into account $\left(E_{C B}=80,000 \mathrm{MPa}\right)$.

\section{COMPUTING STRATEGY}

\subsection{General Overview}

The size of three-dimensional FE meshes, obtained in Section 2.2, requires a large amount of RAM memory to solve the corresponding linear system. In such cases, a common idea is to split the initial problem into subproblems of smaller sizes. This may be achieved by nonoverlapping domain decomposition techniques widely used in structural mechanics and more recently applied to microstructures (Cailletaud et al., 2003). These techniques benefit from the specific architecture of clusters of computers dedicated to parallel computing. Two classes of method are generally considered: balanced domain decomposition (BDD) (Mandel, 1993) and the finite element tearing and interconnecting method (FETI) (Farhat and Roux, 1991). In both methods, an additional problem located at the interface between domains has to be solved in order to ensure global solution compatibility. From a mechanical point of view, the unknowns of the interface problem are displacements in the case of the BDD method and forces for the FETI method. This is the main reason these methods are often referred to as primal and dual methods, respectively. Many works have proved the robustness of the FETI method for FE computations of largescale microstructures, mainly in the case of polycrystals (Barbe et al., 2001; Kanit et al., 2003; Gérard, 2008; Osipov et al., 2008). For these reasons, the FETI method was favored for the microstructures studied in this work. Similarly, many papers deal with the application of FETI on large, highly heterogeneous media (Bhardwaj et al., 1998; Gosselet et al., 2003; Klawonn and Rheinbach, 2006), even though only basic examples are treated therein. 


\subsection{Dual FETI Method for Large Scale Microstructural Computations}

An overview of the FETI techniques in the particular case of structural mechanics is given in detail in Gosselet and Rey (2006). The FETI method is a nonoverlapping domain decomposition method which consists of two successive steps. In the first step, the displacement response of each domain is computed independently. This step results in domains, with non-vanishing relative displacements, the so-called displacement jump. In the second step, the forces needed for the displacement jump at the interface to vanish are computed. For the sake of simplicity, a BVP defined on a volume $\Omega$, subdivided into two domains is considered. The corresponding FE linear system reads

$$
\mathbb{K} \underline{\boldsymbol{u}}=\underline{\boldsymbol{f}} \quad \text { with }\left\{\begin{array}{l}
\underline{\boldsymbol{f}}: \text { vector of nodal forces } \\
\underline{\boldsymbol{u}}: \text { vector of nodal diplacements } \\
\mathbb{K}: \text { global stiffness matrix }
\end{array}\right.
$$

The partition of the microstructure into two domains, $\Omega_{1}$ and $\Omega_{2}$, leads to the following system:

$$
\left(\begin{array}{cc}
\mathbb{K}_{1} & 0 \\
0 & \mathbb{K}_{2}
\end{array}\right) \cdot\left\{\begin{array}{c}
\underline{\boldsymbol{u}}_{1} \\
\underline{\boldsymbol{u}}_{2}
\end{array}\right\}=\left\{\begin{array}{c}
\underline{\boldsymbol{f}}_{1}+\mathbb{B}_{1}^{t} \underline{\boldsymbol{\lambda}} \\
\underline{\boldsymbol{f}}_{2}+\mathbb{B}_{2}^{t} \underline{\boldsymbol{\lambda}}
\end{array}\right\}
$$

where $\underline{\boldsymbol{u}}_{1}$ and $\underline{\boldsymbol{u}}_{2}$ are the vectors of degrees of freedom in subdomains $\Omega_{1}$ and $\Omega_{2}$, respectively. The matrices $\mathbb{B}_{s}$ are Boolean operators which ensure the field transfer from the domains $\Omega_{s}$ to the interface $\Omega_{1} \cap \Omega_{2}$. The interface problem is written as follows:

$$
\begin{gathered}
\left(\sum_{s=1}^{2} \mathbb{B}_{s} \mathbb{K}_{s}^{-1} \mathbb{B}_{s}^{t}\right) \underline{\boldsymbol{\lambda}}=-\sum_{s=1}^{2} \mathbb{B}_{s} \mathbb{K}_{s}^{-1} \underline{\boldsymbol{f}}_{s} \\
\mathbb{D}=\sum_{s=1}^{2} \mathbb{B}_{s} \mathbb{K}_{s}^{-1} \mathbb{B}_{s}^{t}
\end{gathered}
$$

where $\mathbb{D}$ is the Schur dual complement. The equivalent residual problem enforces the continuity of displacement at the interface between domains:

$$
\mathbb{B}_{1} \underline{\boldsymbol{u}}_{1}+\mathbb{B}_{2} \underline{\boldsymbol{u}}_{2}=\underline{\mathbf{0}}
$$

this problem is solved iteratively using the preconditioned conjugate gradient (PCG) (Shewchuk, 1994). At the $\kappa^{\text {th }}$ iteration, the displacement jump reads

$$
\sum_{s=1}^{2} \mathbb{B}_{s} \underline{\boldsymbol{u}}_{s}^{k}=\mathbb{D} \underline{\boldsymbol{\lambda}}^{k}+\sum_{s=1}^{2} \mathbb{B}_{s} \mathbb{K}_{s}^{-1} \underline{\boldsymbol{f}}_{s}
$$

The condition number of the Schur dual complement in Eq. (22) is of primary importance for the convergence of the interface problem. Indeed, conjugate gradient methods are known to be sensitive with respect to conditioning. In the case of a rubber composite, this issue, as well as the detection of rigid body modes, are assessed in the next subsection.

\subsection{Inherent Difficulties for Highly Heterogeneous Microstructures}

\subsubsection{Condition Number of Dual Schur Complement Matrix}

As mentioned in the previous subsection, the condition number of the Schur dual complement is of major importance for the convergence of the interface problem with iterative solvers. With a view to improving this condition number, the use of preconditioners is mandatory. The optimal choice is the Dirichlet preconditioner. Under certain conditions, the lumped preconditioner can achieve a slower convergence of the FETI interface problem. The conditioning of $\mathbb{D}$ is related to the conditioning of each $\mathbb{K}_{s}$. Thus, the decomposition in homogeneous domains (same material) leads 
to the best conditioning number of the Schur dual complement for any preconditioner, including the lumped one. This approach has been successfully tested on a simulated microstructure containing a dozen particles [Fig. 8(a)]. Figure 8(b) shows the corresponding mesh decomposition with homogeneous domains using a dedicated automatic mesher. Here, each domain corresponds to an aggregate or to a part of the matrix. The larger domains can be possibly subdivided like the matrix phase [Fig. 8(b)] or some aggregates [Fig. 9(a)]. For an increasing contrast in elastic properties, the convergence rates were compared to results obtained on the same microstructure partitioned with MetisSplit [Fig. 9(b)]. The contrast is defined as the ratio of Young's modulus of the carbon black particles over Young's modulus of the matrix $\left(E_{C B} / E_{M}\right)$. Contrary to the dedicated automatic mesher, Metis-Split tends to minimize the size of the interface between domains and to create domains containing close numbers of degrees of freedom (11 and 4 domains for the homogeneous domain decomposition and for Metis-Split, respectively). The proposed partitioning is less efficient than Metis-Split when the contrast is lower than $10^{6}$ in the case of KUBC but ensures the convergence for higher contrasts. This method of partitioning creates at least as many domains as aggregates in the simulation and thus cannot be used in the case of too many independent aggregates, for scalability reasons. It can be of interest when the carbon black network percolates in the simulation.

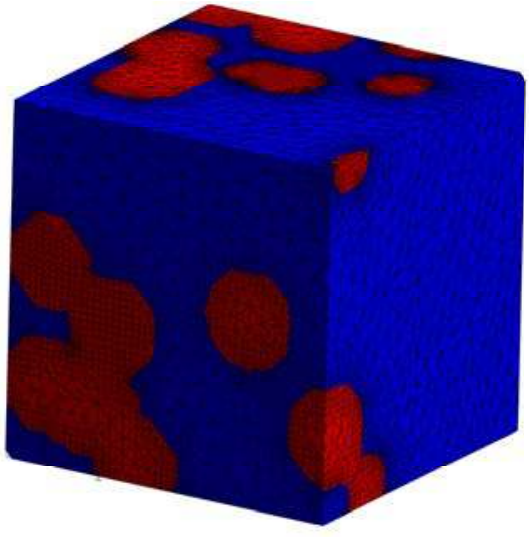

(a)

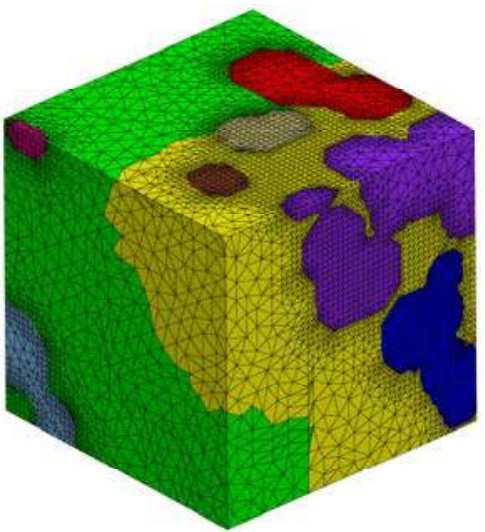

(b)

FIG. 8: (a) Simulation of microstructure containing 12 carbon black particles and (b) decomposition into 11 homogeneous subdomains.

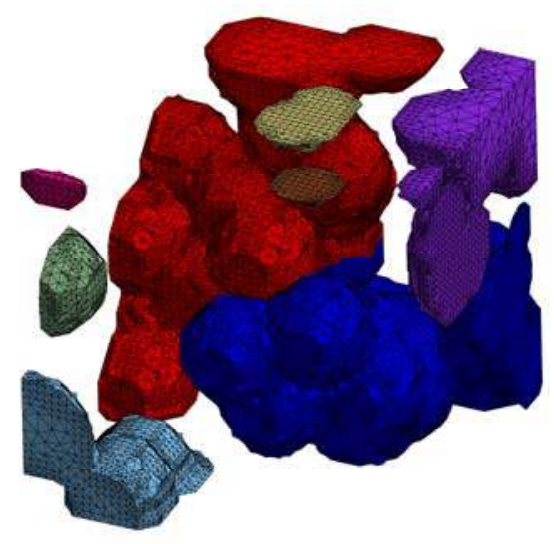

(a)

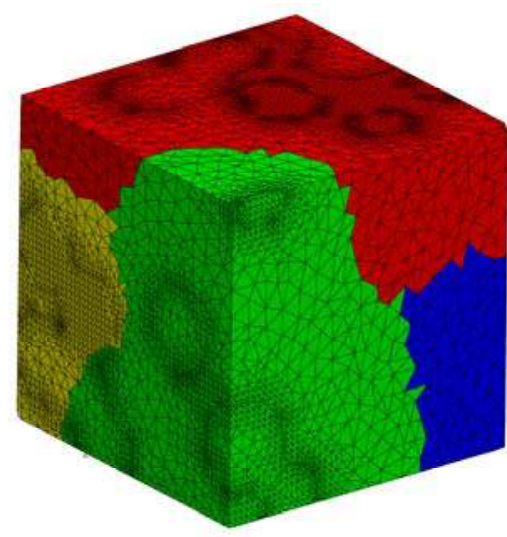

(b)

FIG. 9: (a) Decomposition of the largest aggregate into two subdomains and (b) decomposition into four subdomains using Metis-Split, each color corresponds to one domain. 


\subsubsection{Detection of Rigid Body Modes}

Dealing with mechanics, most local problems, on each domain, are only force-based and thus ill-posed. Their solutions are provided with a linear combination of rigid body modes. Only problems defined on domains located on the global volume boundary where displacements are imposed have a unique solution. In the Z-set environment (MinesParisTech, 2003), the sparse direct solver detects automatically and handles the rigid body modes if necessary. Still, the efficiency of this detection is strongly affected by the contrast between the elastic properties of the constituents and the aspect ratio of the domains. It is recommended to generate domains with an aspect ratio close to one (Bhardwaj et al., 1998). In this work, a strong influence of the geometrical quality of the mesh on the convergence of the FETI problem has also been noticed.

\subsection{Quantitative Study of Convergence Using Uniform Boundary Conditions}

For both uniform boundary conditions, a shear test is performed on a given simulated microstructure. The effect of contrast in terms of the Young's modulus of the phases is investigated. To increase the contrast, the Young's modulus of the carbon fillers is increased from $3 \mathrm{MPa}$ to its current value of $80,000 \mathrm{MPa}$, whereas the Young's modulus of the elastomeric matrix remains constant and equal to $3 \mathrm{MPa}$. Figures 10 and 11 depict the evolution of FETI error as a

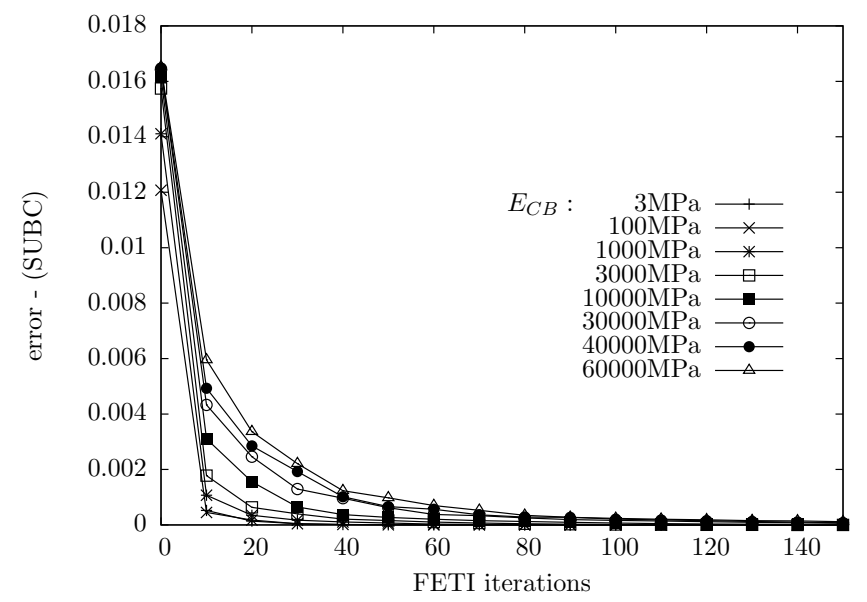

FIG. 10: Influence of elasticity contrast between fillers and matrix on FETI error for SUBC.

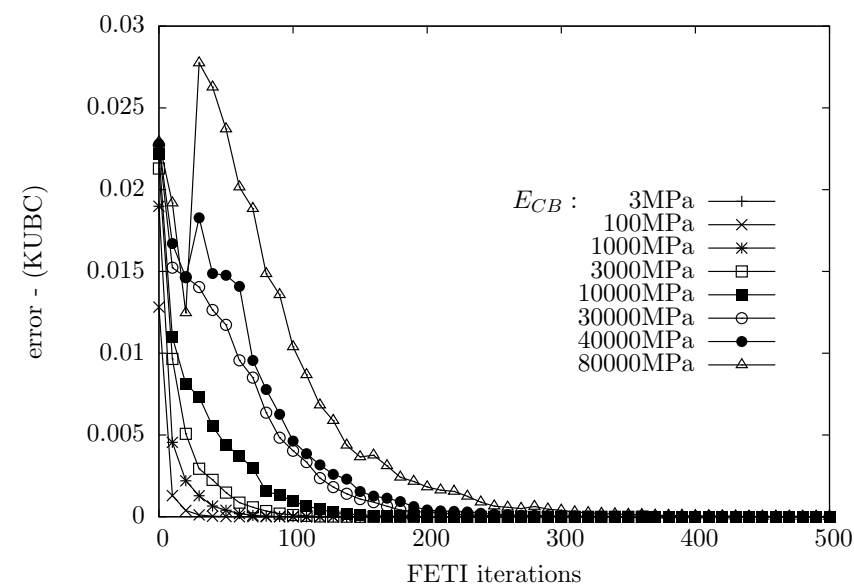

FIG. 11: Influence of elasticity contrast between fillers and matrix on FETI error for KUBC. 
function of the number of iterations for different contrast values in the case of KUBC and SUBC, respectively. The FETI error is the ratio of the displacement jump between subdomains over the initial one. For both cases, the FETI error increases when the Young's modulus of particles increases. Contrary to the KUBC, the use of SUBC does not enable us to reach the real contrast, because the FETI problem finally diverges. Indeed, in the SUBC problem, all the domain problems are ill-posed. Thus, as mentioned in the last subsection, the solver may fail to detect the rigid body modes of each domain because of increasing ill-conditioning due to the larger contrast values. Here, the apparent shear modulus was computed under SUBC while the contrast was increased. Figure 12 shows that the apparent shear modulus reaches an asymptotic value for a contrast ratio larger than 5000, corresponding to a Young's modulus for carbon black particles equal to $15000 \mathrm{MPa}$. This value is retained for the SUBC computations in the determination of RVE size for shear modulus in Section 5.

\subsection{Quasi-Incompressibility of Elastomeric Matrix}

Specific difficulties are encountered when the behavior of the elastomeric matrix is quasi-incompressible. This quasiincompressibility can "lock" the displacements and therefore can increase the apparent elastic moduli of the material. To avoid locking the local displacements, the pressure may be included as a degree of freedom in the FE formulation. Such a mixed formulation (Zienkiewicz and Taylor, 1989) accounts for the possible strong differences of pressure in the material. In the classic FETI method, the interface problem is set to ensuring the displacement continuity between domains. The use of mixed formulation imposes the domain decomposition method to account for the pressure continuity as well. Hybrid domain decomposition methods as proposed in Farhat et al. (2001) ensure the continuity of both primal and dual variables on the interface between domains. They are, thus, also referred as to FETI dual primal (FETI-DP) methods.

In the present work, for linear elasticity, a shear test was performed on the simulated microstructure studied in the last subsection for KUBC and SUBC with a Poisson ratio for the elastomeric matrix that varied from 0.4 to 0.49999. The standard formulation with displacement as a degree of freedom and mixed formulation with displacement and pressure as a degree of freedom were compared. Figure 13 shows the evolution of the apparent shear modulus as a function of the Poisson ratio of the elastomeric matrix for both boundary conditions and for both FE formulations. For values of Poisson ratio very close to 0.5 the standard formulation overestimates the apparent response of material for both boundary conditions (KUBC, SUBC), probably mostly due to the local displacement locking phenomena in the matrix. For a Poisson ratio equal to 0.49 , the mechanical response in linear elasticity is identical for both formulations and remains constant for higher ratios for the mixed formulation. However, it would be meaningless to have the same conclusion for the computation of the bulk modulus directly related to this ratio. The use of a Poisson ratio equal to 0.49 allows use of the classical FETI with only displacement as an unknown in the interface problem. In the following simulations of this work, a Poisson ratio equal to 0.49 was retained to compute the effective shear modulus.

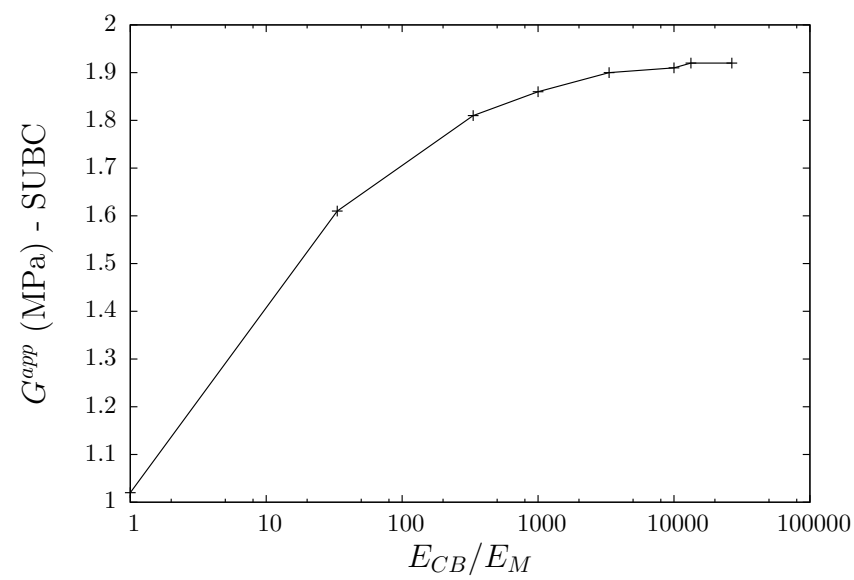

FIG. 12: Influence of the elasticity contrast between fillers and matrix on the apparent response. 


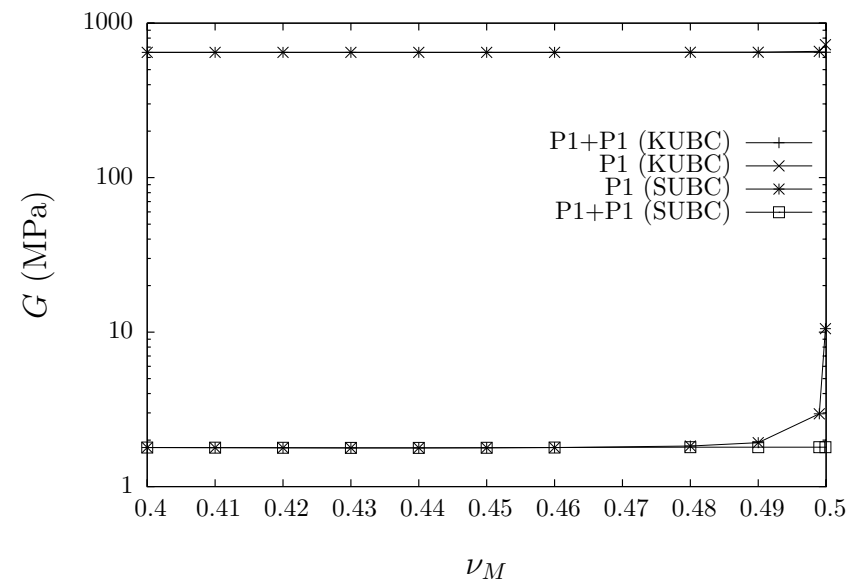

FIG. 13: Apparent shear modulus as a function of Poisson's ratio of elastomeric matrix, influence of the kind of boundary conditions, and FE formulation mixed $(\mathrm{P} 1+\mathrm{P} 1$, linear interpolation of the displacement and of the pressure) and standard (P1, linear interpolation of the displacement).

\subsection{Fast Fourier Transform (FFT) Method}

With no meshing, the FFT (Moulinec and Suquet, 1994) is an alternative approach for computing the local elastic response of a heterogeneous material with complex phase geometry. It makes use of the Lippmann-Schwinger equations:

$$
\begin{aligned}
& \underline{\mathcal{E}}(\underline{\boldsymbol{x}})=\underline{\boldsymbol{\varepsilon}}_{0}-\int_{V} \mathrm{~d} \underline{\boldsymbol{x}}^{\prime} \underline{\sim}^{(0)}\left(\underline{\boldsymbol{x}}-\underline{\boldsymbol{x}}^{\prime}\right): \underline{\boldsymbol{\tau}}\left(\underline{\boldsymbol{x}}^{\prime}\right) \\
& \underline{\boldsymbol{\tau}}(\underline{\boldsymbol{x}})=\underline{\boldsymbol{\sigma}}(\underline{\boldsymbol{x}})-{\underset{\sim}{\boldsymbol{c}}}^{(0)}: \mathfrak{\varepsilon}(\underline{\boldsymbol{x}})
\end{aligned}
$$

where $\underset{\sim}{\boldsymbol{c}^{(0)}}$ is an arbitrary “reference" elastic tensor, $\underset{\widetilde{\sim}}{\boldsymbol{G}}(0)$ is Green's function (of zero average) associated to ${\underset{\sim}{\boldsymbol{c}}}^{(0)}$, and $\underset{\tau}{\widetilde{\tau}}$ is the polarization field. The constant tensor $\underline{\underline{E}}_{0}$ is used to apply a macroscopic (i.e., average) strain loading in the volume $V$. Periodic boundary conditions are assumed (Section 2.2), and $V$ is seen as an elementary cell of the microstructure.

Iterating Eqs. (24) in the real space and (23) in the Fourier domain gives the following fixed-point algorithm, where convergence is measured in terms of stress equilibrium:

$$
\text { set } \underline{\mathfrak{\varepsilon}}^{(0)}(\underline{\boldsymbol{x}}) \equiv \underline{\boldsymbol{\varepsilon}}_{0}, \quad i=0,
$$

iterate

$$
\begin{aligned}
& \underline{\boldsymbol{\tau}}^{(i)}(\underline{\boldsymbol{x}})=\underline{\boldsymbol{\sigma}}^{(i)}(\underline{\boldsymbol{x}})-{\underset{\sim}{\boldsymbol{c}}}^{(0)}: \underline{\boldsymbol{\varepsilon}}^{(i)}(\underline{\boldsymbol{x}}), \quad \underline{\boldsymbol{\sigma}}^{(i)}(\underline{\boldsymbol{x}})=\underset{\sim}{\boldsymbol{c}}(\underline{\boldsymbol{x}}): \underline{\underline{\varepsilon}}^{(i)}(\underline{\boldsymbol{x}}) \\
& \stackrel{\boldsymbol{\tau}}{ }^{(i)}(\boldsymbol{q})=\operatorname{FFT}\left(\underline{\tau}^{(i)}(\underline{\boldsymbol{x}}) ; \boldsymbol{q}\right) \\
& \varepsilon^{(i+1)}(\boldsymbol{q})=-{\underset{\sim}{\boldsymbol{G}}}^{(0)}(\boldsymbol{q}): \underline{\boldsymbol{\tau}}^{(i)}(\boldsymbol{q}), \quad \boldsymbol{q} \neq 0, \quad \underline{\underline{\varepsilon}}^{(i+1)}(\boldsymbol{q}=0)=\varepsilon_{0}
\end{aligned}
$$

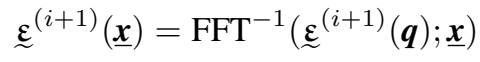

$$
\begin{aligned}
& i=i+1
\end{aligned}
$$

until

$$
\left|q_{i} \sigma_{i j}(\boldsymbol{q})\right|<\eta|\underset{\sigma}{\sigma}(\boldsymbol{q}=0)|
$$


where $\boldsymbol{q}$ are the Fourier modes and $\eta$ is the required precision. Typically in our computations, $\eta=10^{-5}$. According to this procedure, the admissibility of the strain field is enforced at each step, and the displacement field $\mathbf{u}$ is given in the Fourier domain as

$$
u_{i}(\boldsymbol{q})=\frac{2 q_{k} \varepsilon_{i k}(\boldsymbol{q})-3 q_{i} \varepsilon_{m}(\boldsymbol{q})}{|\boldsymbol{q}|^{2}}
$$

where $\varepsilon_{m}=\varepsilon_{i i} / 3$.

Random samples of the mathematical model considered in this work are discretized on a cubic lattice grid to generate microstructure images, of size $L \times L \times L$ voxels, with $L=200,350$, or 500 (where the size of a voxel is about $3.2 \mathrm{~nm}$ ). Prior to discretization, the mathematical model random samples are "periodized," i.e., particles that cut through one of the elementary cell's face (or respective corner) are duplicated, with copies placed along its opposite face (or respective corner) to enforce the microstructure periodic nature. Such treatment leads to an additional volume fraction of particles, for instance, when $L=500$, the volume fraction grows from $16.1 \%$ to $16.9 \%$. To correct this, the effective elastic moduli are corrected proportionally to the volume fraction difference.

For highly contrasted composites such as the material considered in this work, much faster convergence is achieved using the "augmented Lagrangian" algorithm (Michel et al., 2000). This refined algorithm has been used here for this reason. Although the convergence rate of Michel's method highly depends on the choice of the reference tensor ${\underset{\sim}{c}}^{(0)}$, it is generally observed that the optimal reference tensor varies slowly with the size of the microstructure. Accordingly, the reference tensor is numerically optimized for systems containing $64^{3}$ voxels and then used on larger grids.

Contrary to the boundary conditions used in the FFT algorithm, the microstructure model developed here is not periodic. This discrepancy is studied by considering two methods of discretization, referred to as "discontinuous" and "continuous" models, that are detailed below. In the "continuous" model, each random sample is periodized prior to discretization on the voxels grid. More precisely, every spherical inclusion $s_{i}$ is replaced with an infinite set of spheres $\left\{s_{i}+\left(u_{x} \mathbf{e}_{x}+u_{y} \mathbf{e}_{y}+u_{z} \mathbf{e}_{z}\right) L\right\}$ where $u_{x}, u_{y}, u_{z}$ are integers. Although such a model is consistent with PC, it also increases the volume fraction of spheres, and as a consequence of additional sphere connections, greatly decreases the number of aggregates (Table 1). To avoid this drawback, a "discontinuous" discretization is considered, where the periodization step is omitted, i.e., spheres located along the microstructure frontiers are cut along the cube faces. Such a microstructure model is closer to that used by the FEM.

Although the difference between the two models are boundary layer effects, the density of aggregates is very different for the two microstructures, even at large systems sizes (Table 1). As the volume fraction is close to the percolation threshold, a comparison of the elastic response of the two models is necessary to quantify these effects.

\section{RESULTS}

\subsection{Effective Shear Modulus}

Following the procedure proposed by Kanit et al. (2003), the effective shear modulus for a rubber with $14 \%$ of CB fillers was estimated by computing apparent moduli on simulated microstructures of different sizes. Several realizations per size are considered to determine the deviation of the mean value. For a large enough volume element,

TABLE 1: Number of particles and of aggregates (lines 2 and 3, respectively) in microstructures, as used in FFT computations and increasing system sizes (columns 2, 3, and 4). Each number represents one microstructure sample. The number of aggregates depends on the discretization method, "continous" or "discontinuous" (numbers between round and square brackets, respectively)

\begin{tabular}{|c|c|c|c|}
\hline$L(\mathbf{n m})$ & $\mathbf{6 4 0}$ & $\mathbf{1 1 2 0}$ & $\mathbf{1 6 0 0}$ \\
\hline Nb. of particles & 1310 & 7174 & 17951 \\
\hline Nb. of aggregates & {$[141](196)$} & {$[555](723)$} & {$[1841](2170)$} \\
\hline
\end{tabular}


the computed shear modulus converges to the effective response and coincides for any type of boundary condition. For intermediate volumes, the effective shear modulus is estimated from the mean value computed from an adequate number of realizations. Hereby, three sizes of simulated microstructures were considered, corresponding to cubes with edge of 600,800 and $1000 \mathrm{~nm}$ length, respectively. Due to the difficulty to mesh and compute such simulated microstructures, the study was limited to two realizations per size; hence six microstructures were computed. When a given simulation volume element is subjected to a shear test with respect to the three cube directions, the apparent behavior reads

$$
\left(\begin{array}{c}
- \\
- \\
- \\
\Sigma_{12} \\
\Sigma_{23} \\
\Sigma_{31}
\end{array}\right)=\left[\begin{array}{cccccc}
- & - & - & - & - & - \\
- & - & - & - & - & - \\
- & - & - & - & - & - \\
- & - & - & C_{44}^{a p p} & - & - \\
- & - & - & - & C_{55}^{a p p} & - \\
- & - & - & - & - & C_{66}^{a p p}
\end{array}\right]\left(\begin{array}{c}
- \\
- \\
- \\
2 E_{12} \\
2 E_{23} \\
2 E_{31}
\end{array}\right)
$$

The components of stress tensor and strain tensor are given for the SUBC and the KUBC, respectively. For instance, in the case of KUBC the apparent shear moduli $C_{44}^{a p p}$ is estimated by the computed value $\Sigma_{12}$ for $E_{12}=0.5$ and other vanishing strain components. The same method was applied to $C_{55}^{a p p}$ and $C_{66}^{a p p}$ considering the three directions of shear loading $(\underline{\boldsymbol{x}}, \underline{\boldsymbol{y}}),(\underline{\boldsymbol{y}}, \underline{\boldsymbol{z}})$, and $(\underline{\boldsymbol{x}}, \underline{\boldsymbol{z}})$. For each realization of the volume element, the apparent shear modulus was defined by averaging the three components of the fourth-rank tensor $\underset{\sim}{C^{a p p}}$ :

$$
G^{a p p}=\frac{1}{3}\left(C_{44}^{a p p}+C_{55}^{a p p}+C_{66}^{a p p}\right)
$$

Therefore, 36 FE computations were carried out (three sizes, two realizations per size, and six FE simulations per realization). For PC with the FFT method there were three computations per realization. Table 2 gives for each FE simulation the number of spheres and aggregates and the number of unknowns (degrees of freedom, dof) of the problem. The FFT enables us to compute larger volume than the FE method.

Figure 14 displays both the mean and the deviation of the apparent shear modulus obtained for all realizations as a function of the size of the simulated microstructure. This size is given in terms of the length of the edge of the cube of each simulation. The three curves present the results of FE and of the FFT simulations for SUBC and PC, respectively. The two curves concerning the FFT results are related to the two methods of periodization presented in Section 4.6, the continuous and the discontinuous ones. The exact volume fraction of carbon black fillers in each simulation was systematically reported. Since the volume size was fixed and the material random, the carbon volume fraction can fluctuate from one realization to another. The high volume fraction $(>20 \%)$ for one of the two realizations of the smallest microstructure has been retained in order to have a percolated $(\mathrm{P})$ network of carbon black fillers in the simulation, contrary to the other simulations.

Figure 14 shows that, in general, the mean value of the shear modulus decreases when the size of simulation increases for the two boundary conditions, SUBC (FE) and PC (FFT). Furthermore, the periodic response is above the SUBC one, systematically. Both responses are also above the lower Hashin-Shtrikman bound (Hashin and Shtrikman,

TABLE 2: Parameters of simulations of microstructures

\begin{tabular}{|c|c|c|c|c|c|c|}
\hline \multirow{2}{*}{$L(\mathbf{n m})$} & \multicolumn{2}{|c|}{} & \multicolumn{2}{c|}{$\mathbf{8 0 0}$} & \multicolumn{2}{c|}{$\mathbf{1 0 0 0}$} \\
\cline { 2 - 7 } & $\mathbf{R 1}$ & $\mathbf{R 2}$ & $\mathbf{R 1}$ & $\mathbf{R 2}$ & $\mathbf{R 1}$ & $\mathbf{R 2}$ \\
\hline Nb of particles & 1364 & 1311 & 2203 & 2241 & 3951 & 4061 \\
\hline Nb of aggregates & 139 & 107 & 255 & 257 & 443 & 309 \\
\hline Nb of dof $\left(\times 10^{6}\right)$ & 2.5 & 2.2 & 3.1 & 3.6 & 4.5 & 4.2 \\
\hline
\end{tabular}




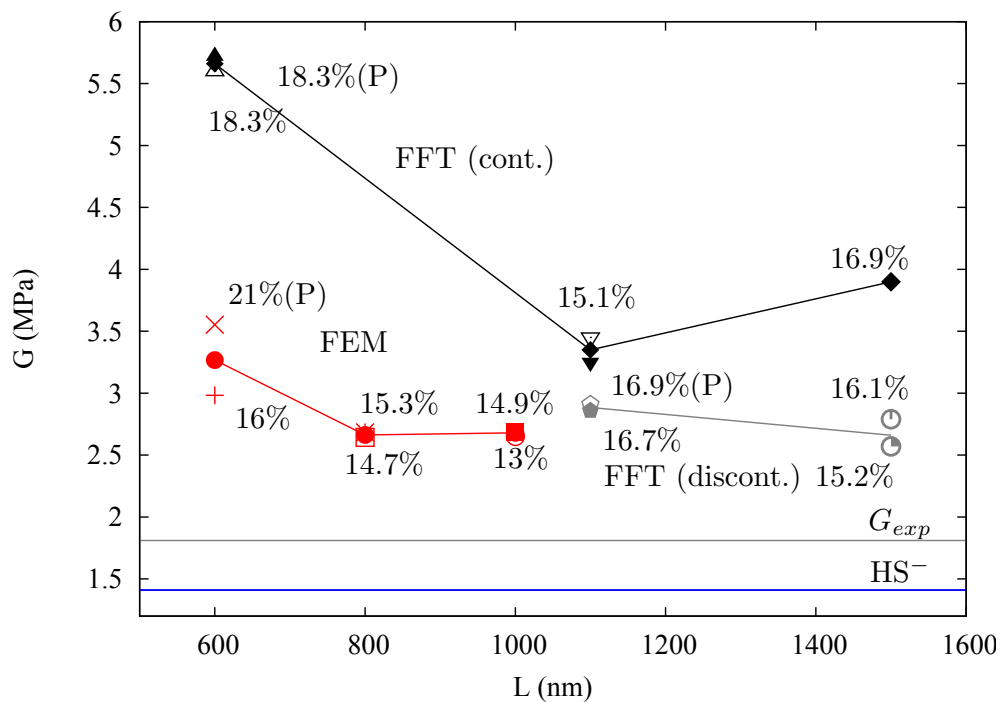

FIG. 14: Apparent shear modulus as a function of the length of the edge of the simulated cube (nm).

1963) and the experimental shear modulus. The volume fraction of most realizations does not reach the volume fraction of the material, equal to $14 \%$. More realizations per size should be considered to obtain the appropriate value by averaging. Nevertheless, a linear interpolation of the mean value of the fraction to $14 \%$ was performed (Fig. 15).

In Fig. 15 the responses for SUBC and PC seem to be stable when increasing the size of generated microstructures. The macroscopic shear modulus, as computed with the FFT method, is larger for "continuous" models than for the "discontinuous" model. Furthermore, an increase of particle connections and decrease of the number of aggregates in the continuous model, compared to the discontinuous model (see Table 1), leads to an increase of the macroscopic shear modulus. Finally, it was found that the elastic response of the discontinuous model, as computed by FFT, is in good agreement with FE computations, and as expected is much closer to the latter than the discontinuous model. The responses may be close to the effective shear modulus.

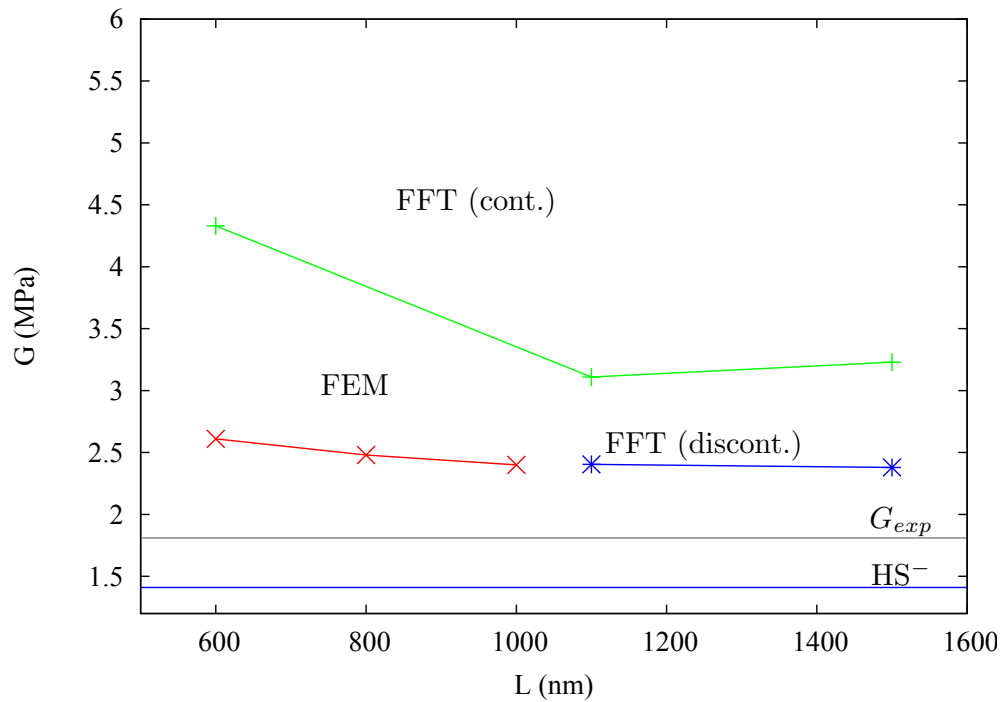

FIG. 15: Apparent shear modulus as a function of domain size $\mathrm{L}(\mathrm{nm})$ for a volume fraction of $14 \%$. Linear interpolation from Fig. 14. 


\subsection{Determination of the RVE Size for the Effective Shear Modulus}

\subsubsection{The Integral Range}

To compute the RVE size (Jeulin, 2005; Kanit et al., 2003), the integral range (Matheron, 1971) was determined from numerical simulations. We consider fluctuations of average values over different realizations of a random medium inside the domain $B$ with volume $V$. In geostatistics, it is well known that for an ergodic stationary random function $Z(x)$, with mathematical expectation $E(Z)$, one can compute the variance $D_{Z}^{2}(V)$ of its average value $\bar{Z}(V)$ over the volume $V$ as a function of the central covariance function $\bar{C}(h)$ of $Z(x)$ (Matheron, 1971) by

$$
D_{Z}^{2}(V)=\frac{1}{V^{2}} \int_{V} \int_{V} \bar{C}(x-y) d x d y
$$

where

$$
\bar{C}(h)=E\{[Z(x)-E(Z)][Z(x+h)-E(Z)]\}
$$

For a sufficiently large specimen, Eq. (26) can be expressed to the first order in $1 / V$ as a function of the integral range in the space $R^{3}, A_{3}$, by

$$
D_{Z}^{2}(V)=D_{Z}^{2} \frac{A_{3}}{V}
$$

with

$$
A_{3}=\frac{1}{D_{Z}^{2}} \int_{R^{3}} \bar{C}(h) d h
$$

where $D_{Z}^{2}$ is the point variance of $Z(x)$ (here estimated from the simulations) and $A_{3}$ is the integral range of the random function $Z(x)$, defined when the integral in Eqs. (26) and (28) is finite. The asymptotic scaling law in expression (27) is valid for an additive variable $Z$ over the region of interest $V$.

To estimate the effective elasticity tensor by simulations, we have to compute the averages $\langle\underset{\sim}{\boldsymbol{\sigma}}\rangle$ for $\mathrm{KUBC}$ or $\langle\underset{\sim}{\varepsilon}\rangle$ for SUBC conditions. For the applied boundary conditions the modulus is obtained from the estimations of a scalar, namel the mean stress or strain field. Therefore the variance of the effective property follows Eq. (27) when the integral range $A_{3}$ of the relevant field is known. Since the theoretical covariance of the fields $(\underset{\sim}{\sigma}$ or $\underset{\sim}{\varepsilon})$ is not available, the integral range can be estimated according to the procedure proposed by Matheron for any random function (Matheron, 1989): working with realizations of $Z(x)$ on domains $B$ with an increasing volume $V$ (or in the present case, considering subdomains of large simulations with a wide range of sizes), we can estimate the parameter $A_{3}$ by fitting the obtained variance according to expression (27). The point variance $D_{Z}^{2}$ of the corresponding field is directly estimated from the numerical variance of the field.

\subsubsection{Practical Determination of the Size of the RVE}

When considering a material as a realization of a random set or of a random function, the idea that there exists one single possible minimal RVE size must be left out. Instead, the size of an RVE can be defined for a physical property $Z$, a contrast of properties, and above all, a given precision in the estimation of the effective property, depending on the number of realizations that one is ready to generate. By means of a standard statistical approach, the absolute error $\epsilon_{a b s}$ and the relative error $\epsilon_{\text {rela }}$ on the mean value obtained with $n$ independent realizations of volume $V$ are deduced from the interval of confidence by

$$
\epsilon_{a b s}=\frac{2 D_{Z}(V)}{\sqrt{n}} \quad ; \quad \epsilon_{\text {rela }}=\frac{\epsilon_{a b s}}{E(Z)}=\frac{2 D_{Z}(V)}{E(Z) \sqrt{n}}
$$

The size of the RVE can now be defined as the volume for which for instance $n=1$ realization is sufficient to estimate the mean property $E(Z)$ with a relative error $\epsilon_{\text {rela }}=1 \%$, provided we know the function $D_{Z}(V)$. Alternatively, we can decide to operate on smaller volumes (provided no bias is introduced by the boundary conditions) and consider $n$ realizations to obtain the same relative error. In the case of an effective elastic moduli, the exact mean value and 
variance for a given domain size are a priori unknown. Using Eq. (29), the absolute error on the mean value can be evaluated. This methodology was applied to the case of the dielectric permittivity of various random media (Delarue, 2001; Jeulin and Moreaud, 2008) and to the elastic properties and thermal conductivity of a Voronoï mosaic (Kanit et al., 2003) or of materials from the food industry (Kanit et al., 2006).

In this work, the largest simulations considered for the statistical study are two volumes of 1000 and $1500 \mathrm{~nm}$ length for SUBC and PC, respectively. For the postprocessing computations, the realization of $1000 \mathrm{~nm}$ length is divided up to 1000 subvolumes $v_{i}$ (Fig. 16). The statistical dispersion of $\sigma_{13}$ for a shear test according to $(\underline{\boldsymbol{x}}, \underline{\boldsymbol{z}})$ axes is observed. Figure 17 illustrates the evolution of the ratio $D_{\sigma_{13}}^{2}(v) / D_{\sigma_{13}}^{2}$ as a function of elementary volume $v$ (or equivalently, the number of domains $N$ in the volume $V, v=V / N)$.

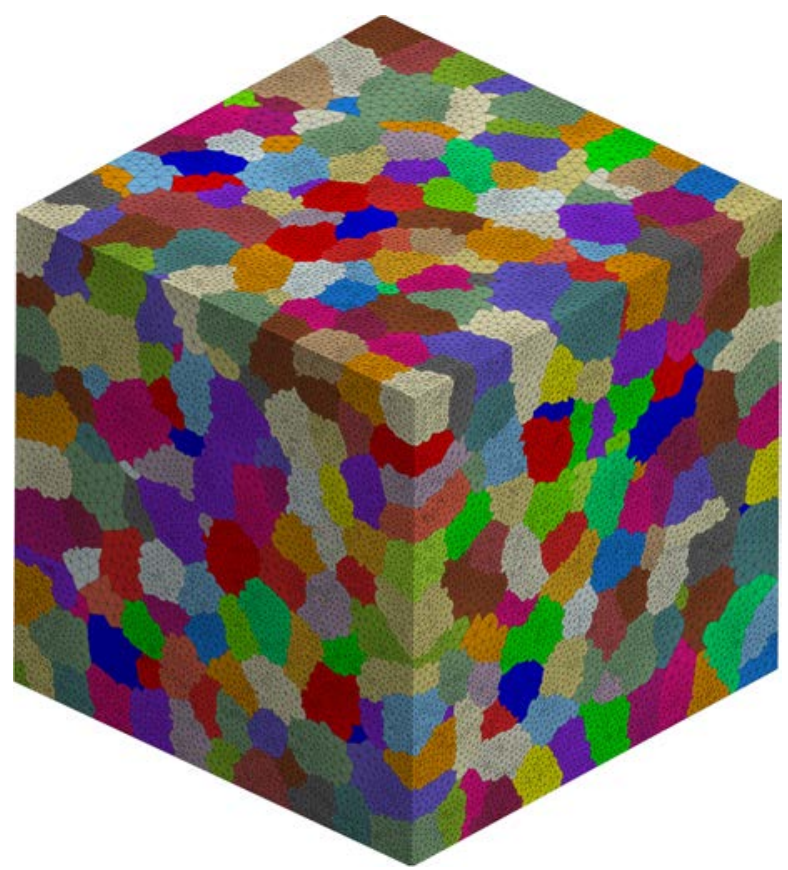

FIG. 16: Decomposition of a microstructure of $1000 \mathrm{~nm}$ edge length into 1000 small volumes.

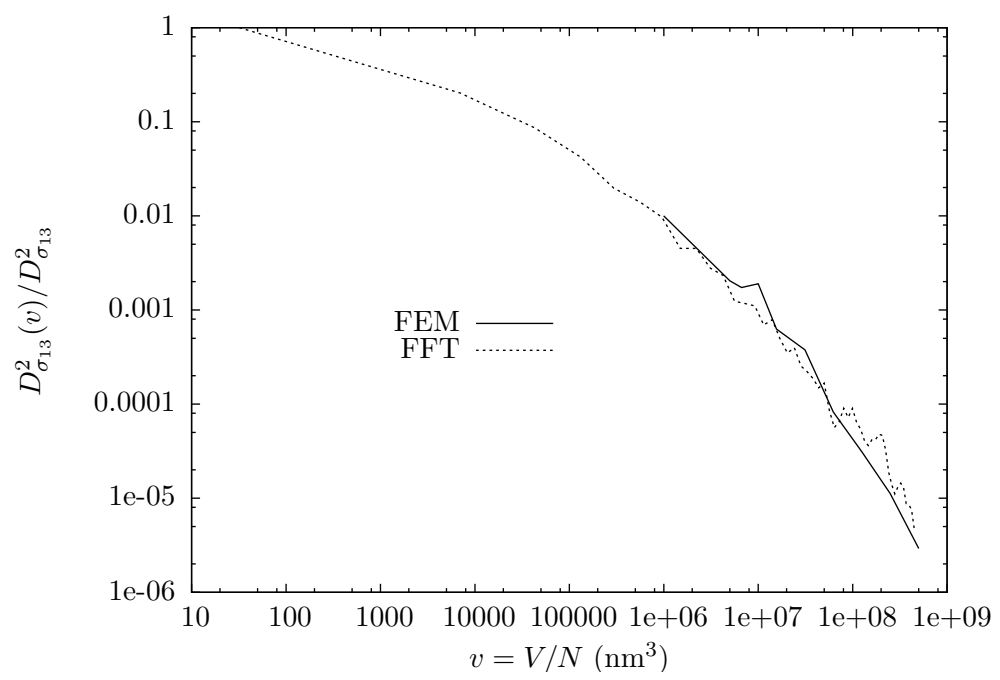

FIG. 17: Variance of apparent mechanical properties on punctual variance as a function of volume of subdomains. 
The ratio $D_{\sigma_{13}}^{2}(v) / D_{\sigma_{13}}^{2}$ decreases when the size of the subvolumes increases, or equivalently, when the number of subvolumes $N$ decreases. The expression (27) is fitted on this curve, for large enough volume $v$, in order to determine the integral range. The obtained integral ranges are equal to $A_{3}^{S U B C}=3.0 \times 10^{7} \mathrm{~nm}^{3}$ and $A_{3}^{P C}=1.06 \times 10^{7}$ $\mathrm{nm}^{3}$ for SUBC and PC, respectively. These values correspond to a cube volume of 998 and $704 \mathrm{~nm}$ of edge length, respectively. In the case of PC, the resulting integral range is smaller than the volume $V$. In the case of SUBC, these two values are very close. In many works (Kanit et al., 2003; Sab and Boumediene, 2005; Hain and Wriggers, 2008) it has been observed that the effective property is reached for smaller volumes in the case of PC than for the other boundary conditions, SUBC, and KUBC. According to the present results, the apparent shear modulus obtained in the case of PC for the simulated microstructures of $1500 \mathrm{~nm}$ edge length may be regarded as the effective property for this rubber filled with $14 \%$ of carbon black particles. Nevertheless, the SUBC response is not far from the PC one. Thus the effective shear modulus for SUBC should be reached for slightly larger volumes.

\subsection{Local Fields}

The local fields were studied in the full volume of a microstructure of $1000 \mathrm{~nm}$ edge length [Fig. 18(a)] and displayed on an extracted slice of a thickness of $100 \mathrm{~nm}$ and with about 30 aggregates which intersect it [Fig. 18(b)].

The first step in the analysis of local fields consisted of looking at stress and strain isovalues in the matrix and in the aggregates (Fig. 19). The histograms of these values were also displayed in the elastomeric matrix to illustrate the field fluctuations. Indeed, very high stress/strain gradients were observed in the matrix. The investigated values are the equivalent von Mises stress and strain:

$$
\left\{\begin{aligned}
\varepsilon_{\text {Mises }} & =\sqrt{\frac{2}{3}\left(\varepsilon_{i j}^{d e v} \varepsilon_{i j}^{d e v}\right)} \\
\sigma_{\text {Mises }} & =\sqrt{\frac{3}{2}\left(\sigma_{i j}^{d e v} \sigma_{i j}^{d e v}\right)}
\end{aligned}\right.
$$

where ${\underset{\sim}{\varepsilon}}^{d e v}$ and $\boldsymbol{\sim}^{d e v}$ denote the deviatioric parts of $\underset{\sim}{\varepsilon}$ and $\underset{\sim}{\sigma}$, respectively. Similarly, the spherical part of the stress and strain tensors are considered:

$$
\left\{\begin{array}{l}
\varepsilon^{S p h}=\operatorname{trace}(\underset{\sim}{\varepsilon}) \\
\sigma^{S p h}=\operatorname{trace}(\underset{\sim}{\sigma})
\end{array}\right.
$$

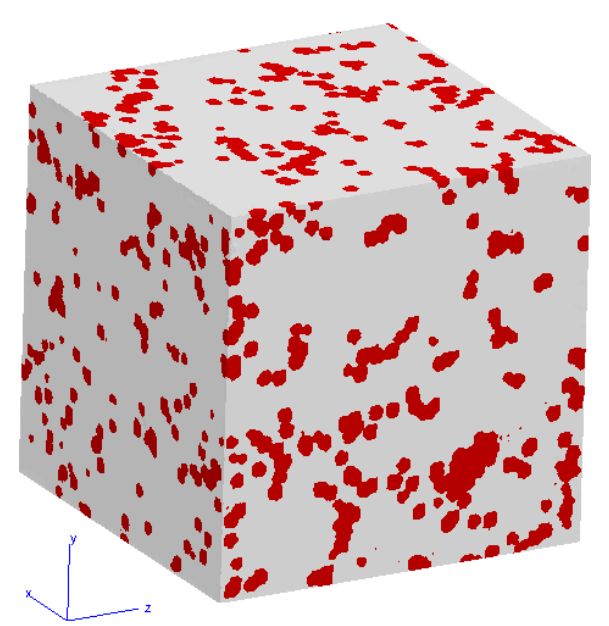

(a)

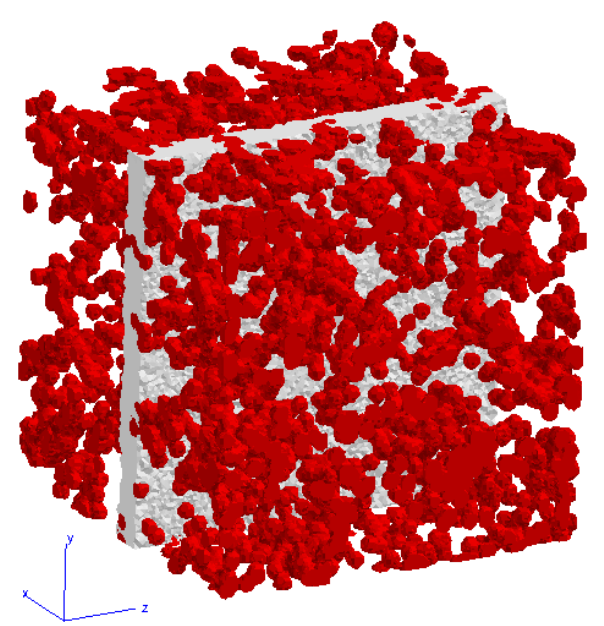

(b)

FIG. 18: The microstructure and the corresponding slice of $100 \mathrm{~nm}$ of thickness considered for the local fields analysis. 


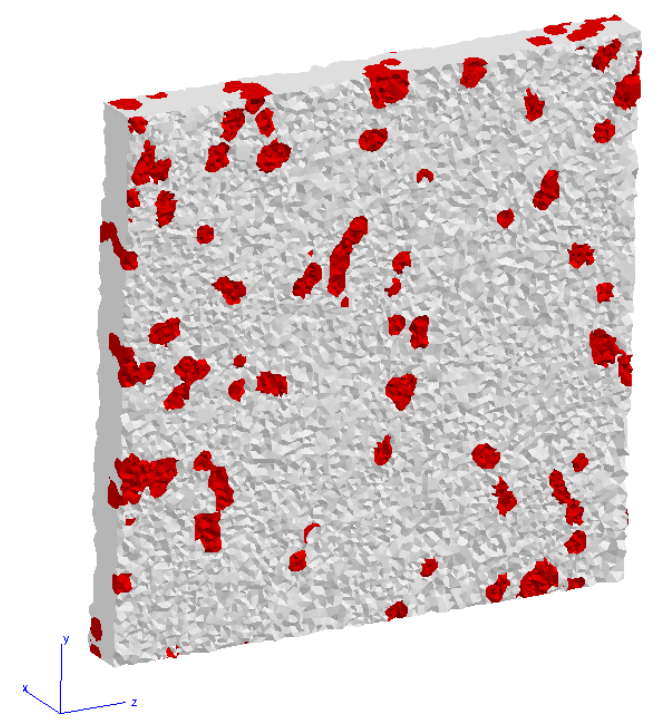

FIG. 19: The aggregates intersecting the studied slice.

Figures 20-23 give the distribution of the trace of stress tensor, the von Mises equivalent stress, the trace of strain tensor (which is proportional to the pressure), and the von Mises equivalent strain. Each distribution is associated with the contour maps in the two phases composing the slice. Table 3 gives the mean value and the standard deviation for each distribution in the elastomeric matrix.

Under SUBC and PC, a shear test leads to a zero global average pressure in the material, or equivalently, to a vanishing average trace of strain tensor, even though large pressures can develop locally inside the material. Figure 22(a) shows the heterogeneous field of the trace of strain tensor in the microstructure due to the interaction of the matrix with aggregates. Figure 22(b) shows the distribution of the trace of strain tensor in the elastomeric matrix alone. A $\chi_{2}$ test has proven that the histogram follows a Gaussian distribution. The trace [Fig. 22(b)] varies from $-0.01 \%$ to $0.01 \%$ for a given shear $\Sigma_{13}=0.1 \mathrm{MPa}$. The positive upper values of the trace are located close to the aggregates. The negative lower values of the trace are located in the matrix far from the aggregates. A study of the trace of the stress tensor (Fig. 20) leads to the same conclusions. Figures 23(a) and 23(b) show the von Mises strain map in the slice and the distribution in the elastomeric matrix, respectively. For most cases, the elastomeric matrix around the aggregates has a very small value of equivalent strain. The mean value of equivalent strain over the matrix is equal to $4.53 \times 10^{-2}$. The global value of the equivalent strain is equal to $3.97 \times 10^{-2}$. Thus, the presence of aggregates amplifies the strain in the matrix. It can be studied by means of two indicators, the local amplification factor $A_{\text {local }}$ and the global amplification factor $A_{\text {global }}$ :

TABLE 3: Mean value and standard deviation of the statistical distributions in the matrix for a shear test in the $(\underline{\boldsymbol{x}}, \underline{\boldsymbol{z}})$ direction, $E_{13}=0.1$

\begin{tabular}{|c|c|c|c|}
\hline (In the matrix) & $\operatorname{tr}(\sigma) \mathbf{M P a}$ & $\operatorname{tr}(\varepsilon)$ & $\sigma_{\text {Mises }} \mathbf{M P a}$ \\
\hline Mean value & $3.49 \times 10^{-3}$ & $2.32 \times 10^{-5}$ & $9.12 \times 10^{-2}$ \\
\hline Standard deviation & 0.1 & 0.0017 & 0.02 \\
\hline \hline (In the matrix) & $\epsilon_{\text {Mises }}$ & $\Sigma_{13} \mathbf{M P a}$ & $E_{13}$ \\
\hline Mean value & $4.53 \times 10^{-2}$ & $3.44 \times 10^{-4}$ & $1.71 \times 10^{-4}$ \\
\hline Standard deviation & 0.02 & - & - \\
\hline
\end{tabular}




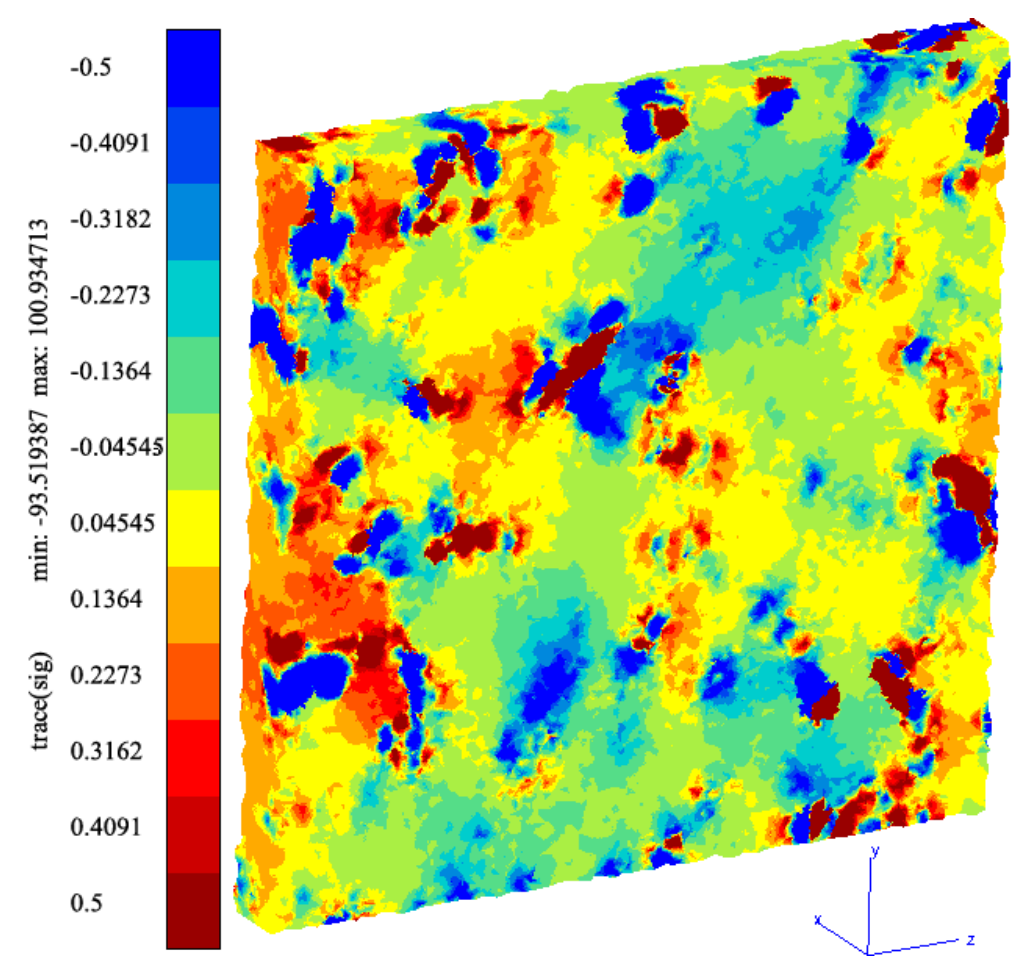

(a)

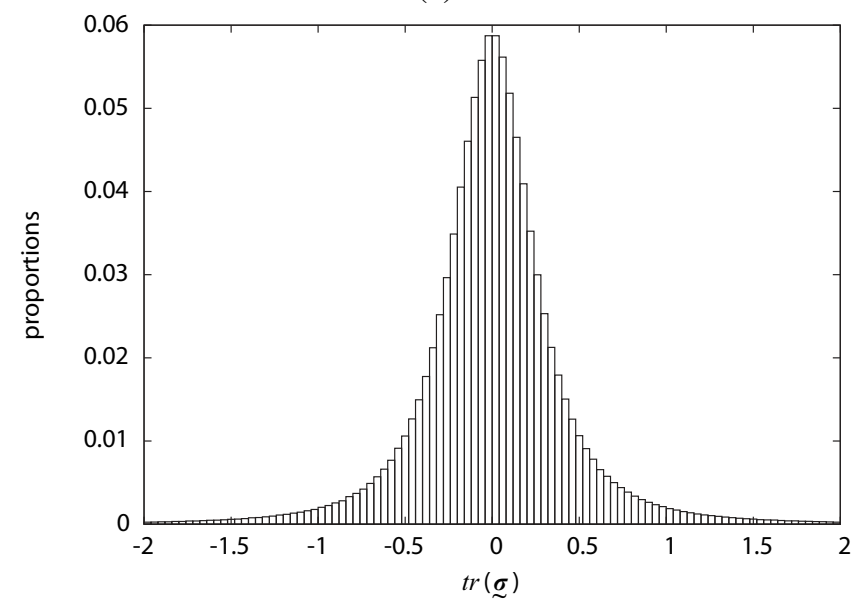

(b)

FIG. 20: Distribution of trace of stress tensor in the matrix component, $\left\langle\sigma^{S p h}\right\rangle_{\text {matrix }}=3.49 \times 10^{-3} \mathrm{MPa}, \Delta[\operatorname{tr}(\sigma)]=$ $0.04 \mathrm{MPa}, \Sigma_{13-\text { prescribed }}=0.1 \mathrm{MPa}$.

$$
A_{\text {local }}(\underline{\boldsymbol{x}})=\frac{\left(\varepsilon_{\text {Mises }}\right)_{\text {matrix }}(\underline{\boldsymbol{x}})}{\left\langle\varepsilon_{\text {Mises }}\right\rangle_{\text {matrix }}} \quad A_{\text {global }}=\frac{\left\langle\varepsilon_{\text {Mises }}\right\rangle_{\text {matrix }}}{\left\langle\varepsilon_{\text {Mises }}\right\rangle_{\text {total }}}
$$

The local amplification was defined at each point belonging to the matrix as the ratio of the local strain over the mean strain of the matrix. (For illustration see Fig. 23, showing the field $\varepsilon_{\text {Mises }}$ in the matrix.) The global amplification was defined as the ratio of the mean strain in the matrix over the mean strain in the entire microstructure. Another definition of the global strain amplification was proposed in Bergstrom and Boyce (1998). This definition corresponds 


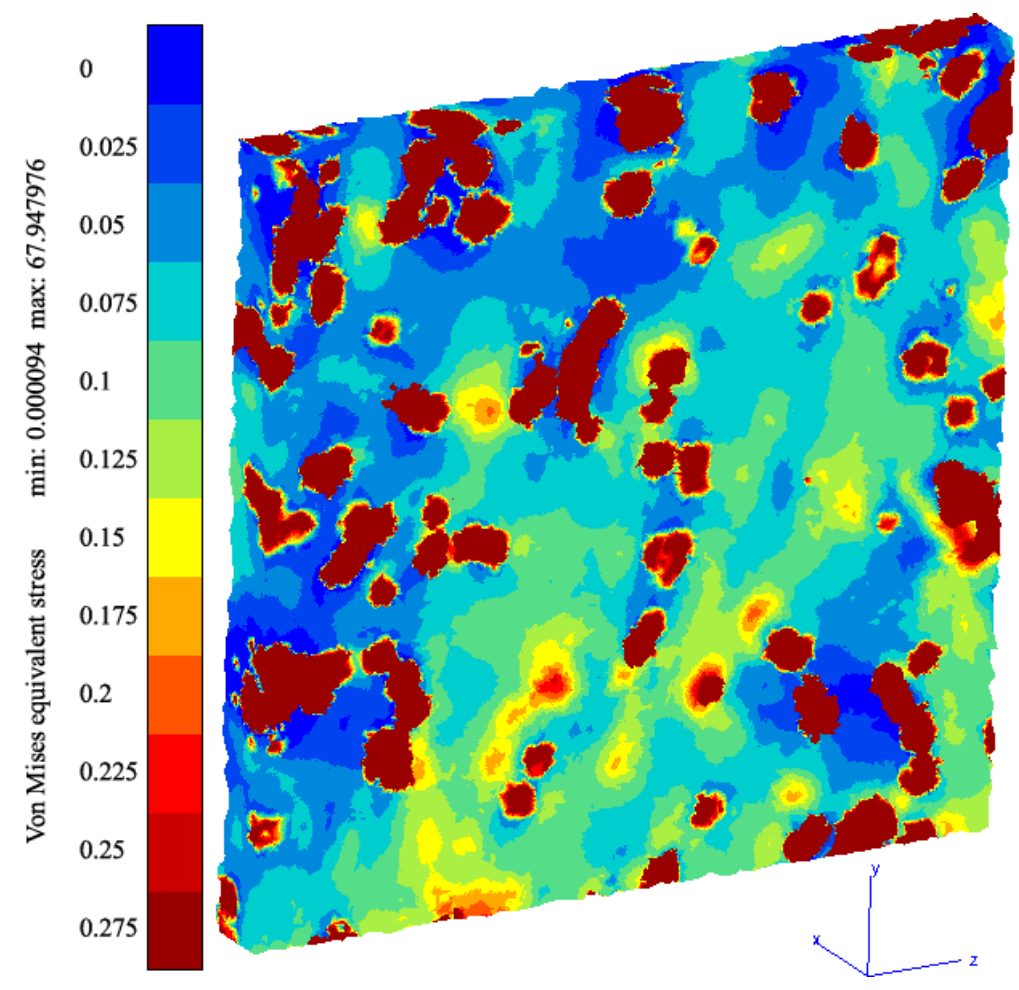

(a)

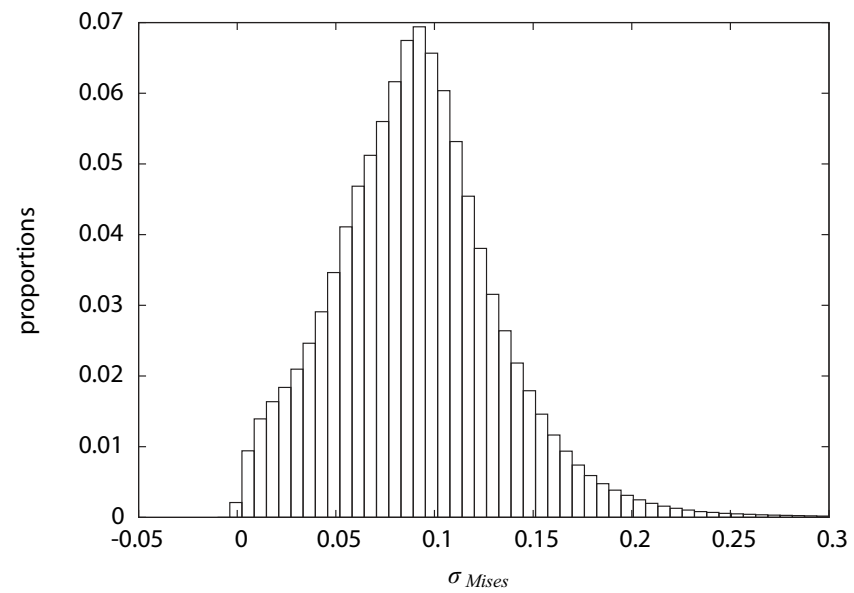

(b)

FIG. 21: Distribution of von Mises equivalent stress in the matrix component, $\left\langle\sigma_{\text {Mises }}\right\rangle_{\text {matrix }}=9.12 \times 10^{-2} \mathrm{MPa}$, $\Delta\left(\sigma_{\text {Mises }}\right)=5.0 \times 10^{-3} \mathrm{MPa}, \Sigma_{13-\text { prescribed }}=0.1 \mathrm{MPa}$.

to the ratio of local strain in the matrix over the strain of the corresponding unfilled material. Here, amplifications are studied in terms of the von Mises strain. The maximum local amplification value is close to 3 . The global amplification value is equal to 1.14. The global amplification is linked to the volume fraction of the fillers. The local amplification is also linked to the volume fraction but mainly linked to the dispersion of particles and aggregates in the matrix. It is interesting to identify the matrix zones where the maximum of local amplification is located. 


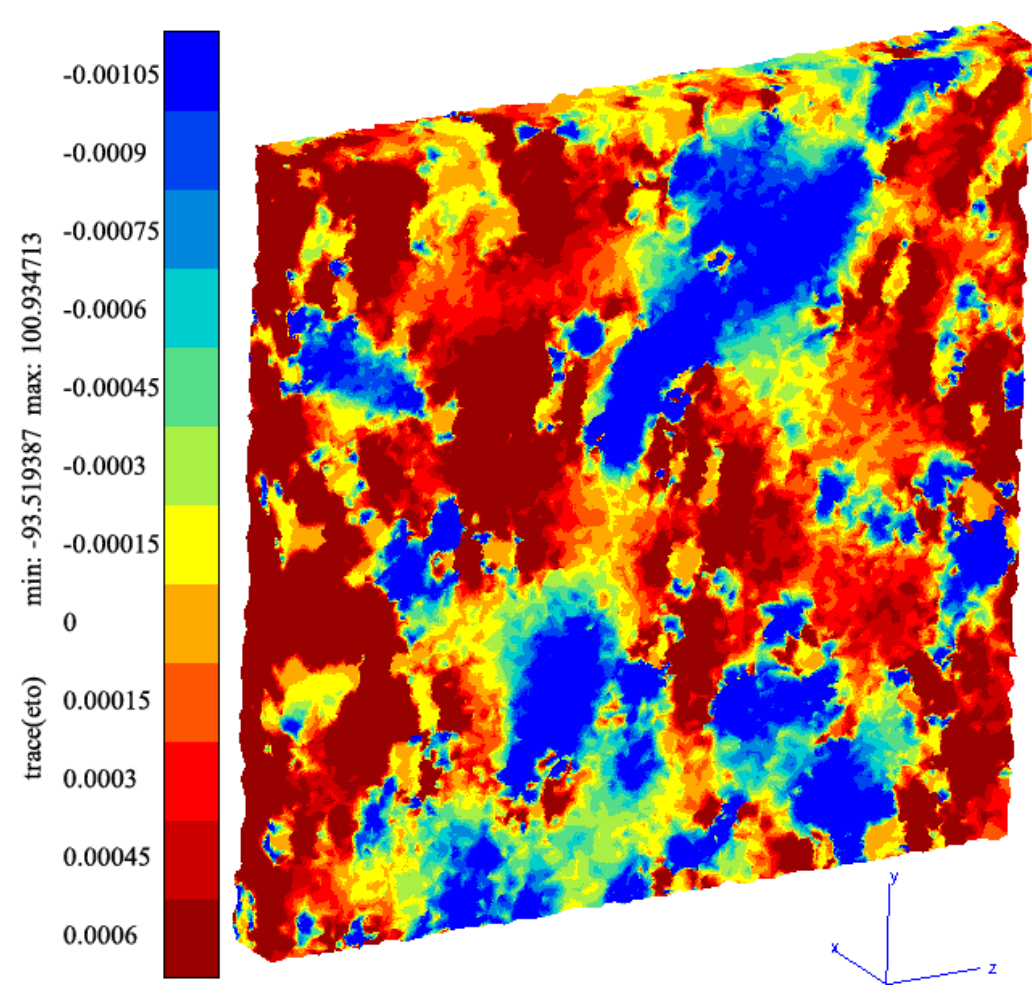

(a)

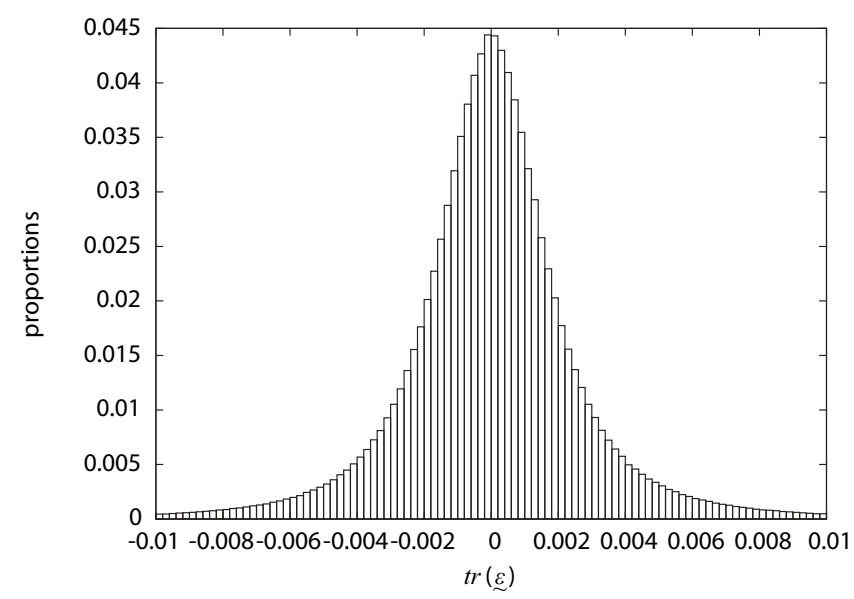

(b)

FIG. 22: Distribution of trace of strain tensor in the matrix component, $\left\langle\varepsilon^{S p h}\right\rangle_{\text {matrix }}=2.32 \times 10^{-5}, \Delta[\operatorname{tr}(\varepsilon)]=$ $2.0 \times 10^{-3}, \Sigma_{13-\text { prescribed }}=0.1 \mathrm{MPa}$.

\section{DISCUSSION}

The interpolated effective shear modulus of simulated microstructures slightly overestimated the experimental data (Table 4). Some possible reasons for that are related to the specific behavior of elastomers. Some works (Leblanc, 2002; Donnet, 2003) assumed that the global mechanical behavior of the composite is mainly affected by the local behavior of the elastomeric matrix located close to aggregates. The elastomeric matrix located near aggregates may 


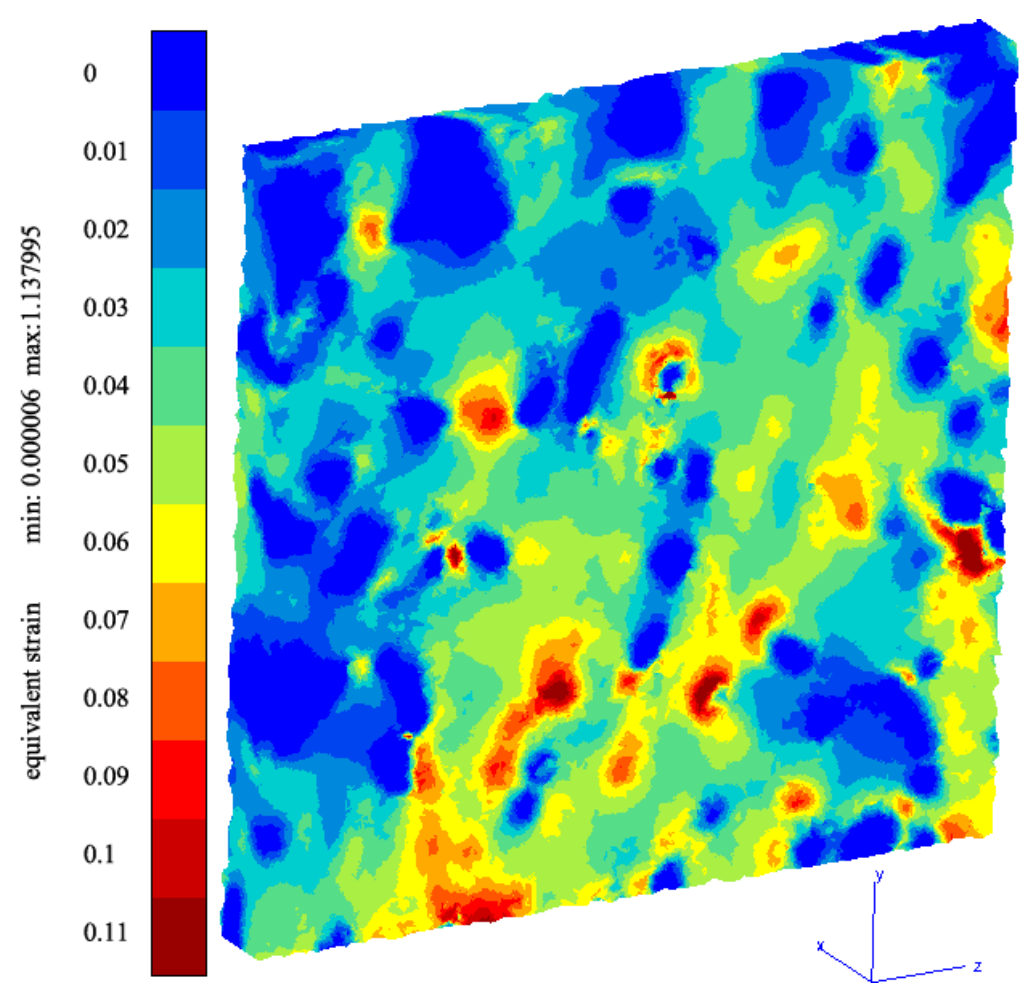

(a)

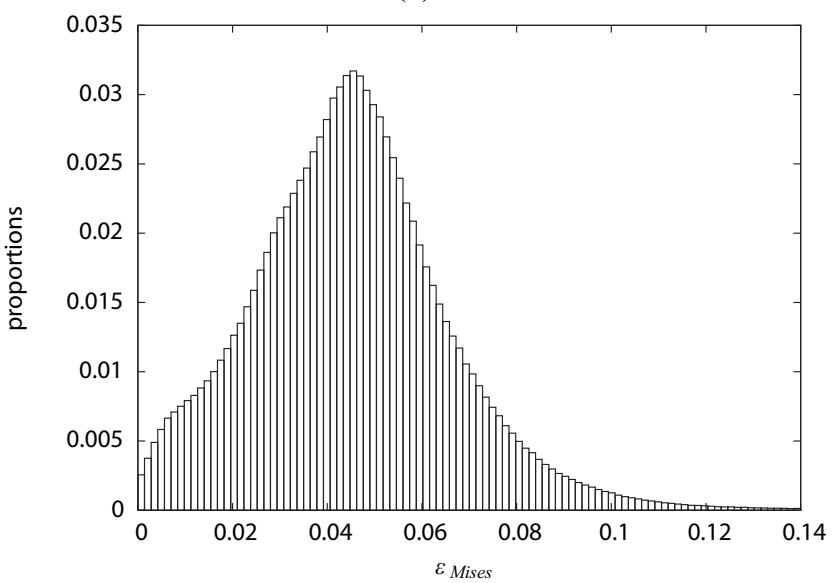

(b)

FIG. 23: Distribution of equivalent strain, $\left\langle\varepsilon_{\text {Mises }}\right\rangle_{\text {matrix }}=4.53 \times 10^{-2}, \Delta\left[\varepsilon_{\text {Mises }}\right]=1.4 \times 10^{-3}, \Sigma_{13-\text { prescribed }}=$ $0.1 \mathrm{MPa}$.

have stiffer properties than the rest of the elastomeric matrix. The size of this bonded rubber is not controlled inside the model, and this size may be overestimated by the FE computation. A third phase may be introduced in this area in the matrix with a particular thickness and physical properties (Marcadon et al., 2007). It is also possible to introduce a thick layer around the carbon black fillers with weakened properties in order to reduce its apparent physical property.

The second possible reason can be related to the identification of the microstructure model. Indeed, a slight oversegmentation of particles in aggregates on TEM images has been noticed. This oversegmentation results in aggregates with more carbon black fillers in binary TEM images than in the experimental ones. The morphological model being 
TABLE 4: Values of interpolated shear modulus to convergence obtained for KUBC and PC, compared to the experimental data and the length corresponding to the integral range

\begin{tabular}{|l|c|c|c|}
\hline & SUBC & PC & Exp \\
\hline$G_{\text {interp }}^{\text {app }}(\mathrm{MPa})$ & 2.4 & 2.4 & 1.81 \\
\hline$L_{A_{3}}(\mathrm{~nm})$ & 998 & 704 & - \\
\hline
\end{tabular}

identified on binary TEM images, the aggregates in the simulated microstructure may contain more particles which are more entangled and thus tend to reinforce the composite. It would be interesting to slightly reduce the volume fraction of particles in the inclusions in order to decrease the intersection volume between particles. This modification has to be performed with an increase of the volume fraction of the inclusions in order to keep the global volume fraction of carbon black fillers constant.

Another material with the same volume fraction of fillers, namely $14 \%$, is studied. During material processing, this material was mixed during twice less time. In order to distinguish this material from the one studied in the last section, they are called $M_{t}$ and $M_{t / 2}$, respectively. Figures 24(a) and 24(b) display the experimental TEM images of the materials $M_{t}$ and $M_{t / 2}$. The carbon black particles and aggregates of the microstructure of the material $M_{t / 2}$ are less dispersed than the ones of $M_{t}$. Figure 25(a) shows a simulated microstructure of $M_{t / 2}$ exhibiting a percolated network of the particles. Figure 25(b) illustrates the largest percolating aggregate. These carbon black fillers are less homogeneously distributed than the material studied in the last subsection, $M_{t}$. A shear test was performed on this simulated microstructure. The ratio, from FE simulations, between the shear modulus for $M_{t}$ and $M_{t / 2}, G_{M_{t}} / G M_{t / 2}$, is the same $(\simeq 10 \%)$ as the one obtained experimentally. Thus the present general method seems to be able to account for the morphological effect associated with material processing. This is not the case of the Hashin-Shtrikman bound, which provides here a good estimate of the experimental property. The results of the prediction of the shear modulus of the two considered materials enable us to give a further interpretation of the overestimation. Due to the conditions of mixing, material $M_{t / 2}$ is expected to create more occluded elastomer inside carbon black aggregates, resulting in a higher modulus. It can be suspected that the morphological model slightly overestimates the fraction of occluded elastomer in both cases, resulting in a higher apparent modulus.

Figure 26 illustrates the two morphological configurations of aggregates in the matrix considered for the local analysis in the slice. The first case represents the field between two aggregates called A and B. The second case considers the field in the matrix between branches of the same aggregate, B. The elastomer located between the aggregates

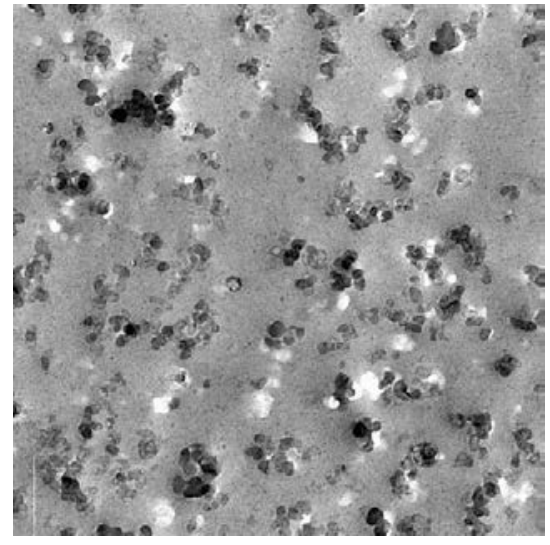

(a)

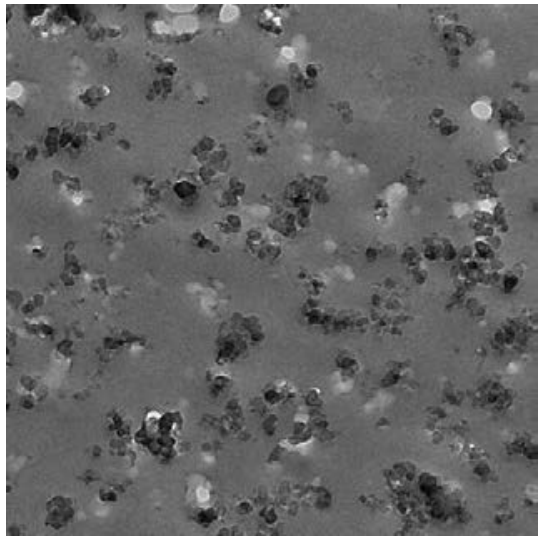

(b)

FIG. 24: TEM images of the material $M_{t}$ (a) and the material $M_{t / 2}$ (b), micrographs of $1500 \mathrm{~nm}$ edge length. 


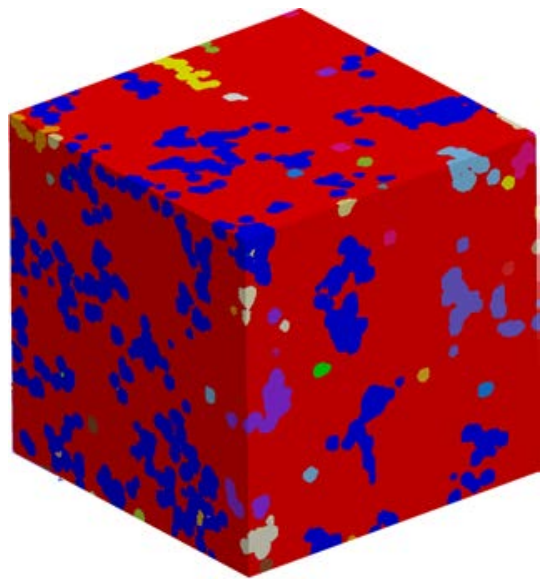

(a)

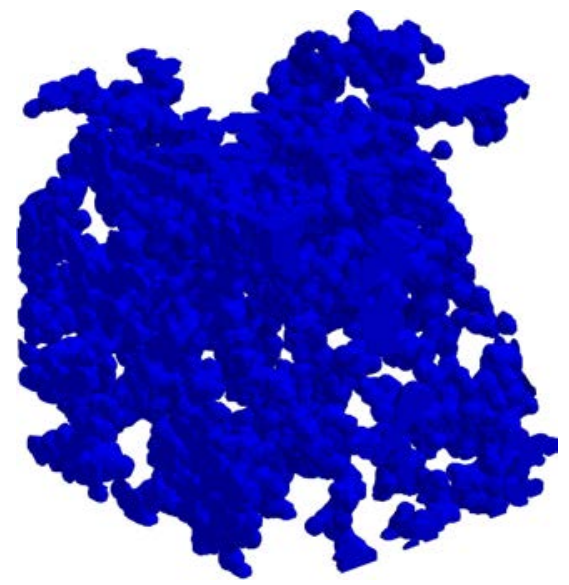

(b)

FIG. 25: Simulation of the material $M_{t / 2}$ differentiating the aggregates (a) and its percolated network (b).
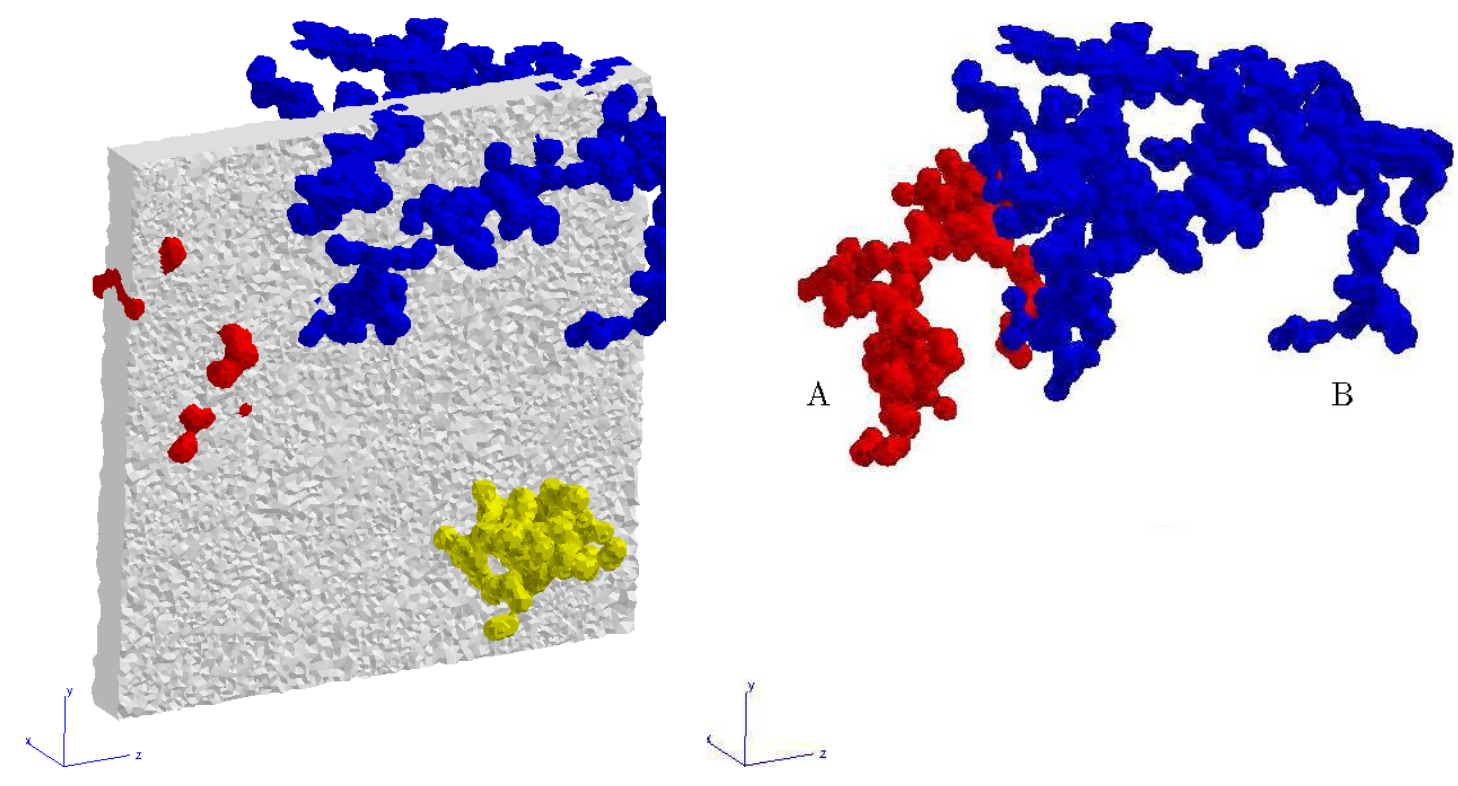

FIG. 26: Aggregates A and B studied in the local analysis of the field.

A and B was subjected to the strongest local strain amplification, approximatively equal to 3 . This phenomenon is due to the difficulty of the rubber matrix to deform its surrounding aggregates. This rubber is located between the branches of aggregates where the matrix is compressed $[\operatorname{tr}(\underset{\sim}{\varepsilon}) \leq 0]$. Indeed, the matrix located between the branches of aggregate $\mathrm{A}$ has a local amplification equal to 0.66 , whereas the matrix located between the branches of aggregate $\mathrm{B}$ has a local amplification equal to 1.33. The matrix located between the branches of aggregate $\mathrm{B}$ is less confined than aggregate $\mathrm{A}$. The structure of the aggregates can definitely have a strong influence on the local mechanical behavior, locally amplifying the strain/stress in the matrix and thus modifying the global stiffness of the composite.

\section{CONCLUSIONS AND PROSPECTS}

A method was proposed in this paper to estimate the apparent/effective elastic properties of a rubber with carbon black nanoparticles from computations of its microstructure. A mathematical model associated with an original method of 
identification was established in order to simulate three-dimensional virtual microstructures. This mathematical model and the method of identification are explained in detail in Jean et al. (2010).

For the FE method, a meshing algorithm was proposed to take the complex geometry of the heterogeneous media into account. The obtained mesh requires domain decomposition techniques for parallel computations. Regarding the highly heterogeneous behavior of the material, special attention was focused on the quality of the three-dimensional mesh and on the influence of the mechanical contrast between phases on the apparent response. Furthermore, in linear elasticity, the shear modulus was computed with a Poisson ratio equal to 0.49 using a standard FE formulation. In contrast, the FFT computations could be performed with the actual value of Poisson ratio in the rubber matrix.

For an isotropic linear elasticity behavior, the apparent shear moduli were computed on simulated microstructures with increasing sizes. Three boundary conditions were used: the KUBC, the SUBC using FE approach, and the PC using the FFT method. From the statistical approach detailed in Kanit et al. (2003), the integral range was determined for the SUBC and periodic conditions. It seems that the RVE is reached in the case of PC, in agreement with the computations in Kanit et al. (2003) and Lachihab (2004). The obtained effective value of the shear modulus overestimates the experimental value. Thus in order to underline the efficiency of the method and the approach, the elastic property of another material associated with another mixing time was computed. The deviation on shear modulus between this material and the initial one is similar to the one obtained experimentally. The present general method is able to account for the influence of the morphology and the material processing.

Finally, an analysis of local fields in the microstructure, submitted to shear loading, was performed in order to characterize the interaction between aggregates and the elastomeric matrix. The local and global strain amplification were studied in the matrix. The matrix located between two aggregates seems to be subjected to an increasing strain amplification when the distance between aggregates decreases.

The next step will be the systematic use of local field analysis to understand the local mechanical phenomena in each phase and between heterogeneities. For instance, the study of strain and stress amplification can be very useful to predict local failure for most of the materials. Another step will be to consider a more realistic rubber behavior in the sense of large deformation loading and high nonlinear mechanical behavior. Numerous difficulties will arise due to the high nonlinearity of the problem, such as in the case of the domain decomposition method for solving the FE problem. The use of domain decomposition usually requires use of a remeshing procedure to maintain an acceptable shape ratio and mesh quality in the domain. Finally, the study of RVE size in the nonlinear context is a challenge for further studies.

\section{ACKNOWLEDGMENTS}

The authors acknowledge the Michelin Research Technology Center for their financial and scientific support.

\section{REFERENCES}

Barbe, F., Decker, L., Jeulin, D., and Cailletaud, G., Intergranular and intragranular behavior of polycrystalline aggregates. Part 1: FE model, Int. J. Plast., vol. 17, pp. 513-536, 2001.

Ben Dhia, H., Multiscale mechanical problems: the Arlequin method, C. R. Acad. Sci., vol. 326, pp. 899-904, 1998.

Beran, M., Statistical Continuum Theory, John Wiley, New York, 1968.

Bergstrom, J. and Boyce, M., Constitutive modelling of the large strain time-dependent behavior of elastomers, J. Mech. Phys. Solids, vol. 46, pp. 931-954, 1998.

Bhardwaj, M., Day, D., Farhat, C., Lesoinne, M., Pierson, K., and Rixen, D., Application of the FETI method to ASCI problemsscalability results on 1000 processors and discussion of highly heterogeneous problems, Int. J. Num. Meth. Eng., vol. 47, pp. 513-535, 1998.

Bruggeman, D., Berechnung verschiedener physikalisher konstante von heterogene substanzen, Ann. Phys., vol. 24, p. $636,1935$.

Cailletaud, G., Forest, S., Jeulin, D., Feyel, F., Galliet, V., Mounoury, V., and Quilici, S., Some elements of microstructural mechanics, Comput. Mater. Sci., vol. 27, pp. 351-374, 2003. 
Cantournet, S., Desmorat, R., and Besson, J., Mullins effect and cyclic stress softening of filled elastomers by internal sliding and friction thermodynamics model, Int. J. Solids Struct., vol. 46, pp. 2255-2264, 2009.

Delarue, A., Prévision du Comportement Électromagnétique de Matériaux Composites Partir de Leur Mode d'Élaboration et de Leur Morphologie, PhD Thesis, École des Mines de Paris, 2001.

Diani, J., Fayolle, B., and Gilormini, P., A review on the Mullins effect, European Polymer Journal, vol. 45, pp. 601-612, 2009.

Donnet, J., Nano and microcomposites of polymers elastomers and their reinforcement, Compos. Sci. Technol., vol. 63, pp. 10851088, 2003.

Farhat, C., Lesoinne, M., LeTallec, P., Pierson, K., and Rixen, D., FETI-DP: a dual-primal unified FETI method-Part I: a faster alternative to the two-level FETI method, Int. J. Numer. Meth. Eng., vol. 50, pp. 1523-1544, 2001.

Farhat, C. and Roux, F. X., A method of finite element tearing and interconnecting and its parallel solution algorithm, Int. J. Numer. Meth. Eng., vol. 32, pp. 1205-1227, 1991.

Feyel, F. and Chaboche, J., $\mathrm{FE}^{2}$ multiscale approach for modeling the elastoviscoplastic behavior of long fibre sic/ti composite materials, Comput. Meth. Appl. Mech. Eng., vol. 183, pp. 309-330, 2000.

Frey, P. and Georges, P., Mesh Generation-Application To Finite Elements, HERMES, Paris, 1999.

Georges, P. and Borouchaki, H., Triangulation de Delaunay et Maillage, Applications aux Éléments Finis, HERMES, Paris, 1997.

Gérard, C., Mesures de Champs et Identification de Modèles de Plasticité Cristalline, PhD thesis, Université Paris XIII, 2008.

Gérard, C., N'Guyen, F., Osipov, N., Cailletaud, G., Bornert, M., and Caldemaison, D., Comparison of experimental results and finite element simulation of strain localization scheme under cyclic loading, Comput. Mater. Sci., vol. 46, pp. 755-760, 2009.

Gosselet, P. and Rey, C., Non-overlaping domain decomposition methods in structural mechanics, Arch. Comput. Meth. Eng., vol. 13, pp. 515-572, 2006.

Gosselet, P., Rey, C., and Rixen, D., Etude comparative des méthodes de décomposition de domaine primal et duale: Vers une meilleure initialisation de FETI, Nice, France, 16ème Congrés Francais de Mécanique, 2003.

Govindjee, S. and Simo, J. C., Mullins effect and the strain amplitude dependence of the storage modulus, Int. J. Solids Struct., vol. 29, pp. 1737-1751, 2009.

Hain, M. and Wriggers, P., Computational homogenization of microstructural damage due to frost in hardened cement paste, Int. J. Numer. Meth. Eng., vol. 52, pp. 139-160, 2008.

Hashin, Z. and Shtrikman, S., A variational approach to the theory of the elastic behavior of multiphase materials, J. Mech. Phys. Solids, vol. 11, pp. 127-140, 1963.

Holownia, B., Effect of carbon black on the elastic constants of elastomers, Rubber Industry, pp. 157-160, 1974.

Huet, C., Application of variational concepts to size effects in elastic heterogeneous bodies, J. Mech. Phys. Solids, vol. 8, pp. 813-841, 1990.

INRIA, Institut national de recherches en informatique et automatique home page, http://www.inria.fr/, 2009.

Ionescu, I., Moës, N., Cartraud, P., Chevaugeon, N., and Béringhier, M., Eds., Image-based Michromechanics Analysis Using Level Sets and the Extended Finite Elements Method, Ecole des Mines de Paris. IWCCM17, 2007.

Jean, A., Etude d'un Élastomère Chargé, de sa Nanostructure à Son Macro-Comportement, PhD Thesis, Ecole des Mines de Paris - ParisTech, 2009.

Jean, A., Jeulin, D., Cantournet, S., Forest, S., and N'Guyen, F., Rubber with carbon black fillers: Parameters identification of a multiscale nanostructure model, In Boukamel, A., Laiarinandrasana, L., Meo, S., and Verron, E., Eds., Proc. $5^{\text {th }}$ European Conf. Consitutive Models for Rubber, ECCMR 2007, Paris, France, 4-7 Sept. 2007, pp. 141-146, London, Taylor and Francis, 2007.

Jean, A., Jeulin, D., Forest, S., Cantournet, S., and N'Guyen, F., A multi-scale microstructure model of carbon black distribution in rubber, J. Microsc., vol. 241, pp. 243-260, 2010.

Jeulin, D., Modèles Morphologiques de Structures Aléatoires et de Changement d'Échelle, Thèse de Doctorat d'Etat, Université de Caen, France, 1991.

Jeulin, D., Random Structures in Physics, in: Space, Structure and Randomness, Contributions in Honor of Georges Matheron in the Fields of Geostatistics, Random Sets, and Mathematical Morphology. Lect.Notes Statistics, vol. 183, no. XIV, pp. 183-222, 2005. 
Jeulin, D. and Le Coënt, A., Morphological modeling of random composites in continium models discrete system, In ENSMP, Ed., 8th Int. Symp., Varna, Bulgarie, pp. 199-206, Fontainebleau, 1995.

Jeulin, D. and Moreaud, M., Statistical representative volume element for predicting the dielectric permittivity of random media, pp. 429-436, 2008, Presses de l'Ecole des Mines de Paris. CMDS 11 (Paris, 30 July-3 August 2007).

Jeulin, D. and Ostoja-Starzewski, M., Mechanics of Random and Multiscale Microstruct., Springer, Wien, NewYork, 2001.

Jha, V., Thomas, A., Fukahori, Y., and Busfield, J., Micro-structural finite element modelling of the stifness of filled elastomers: the effect of filler number, shape and position in the rubber matrix, In Boukamel, A., Laiarinandrasana, L., Meo, S., and Verron, E., Eds., Proc. $5^{\text {th }}$ Eur. Conf. Consitutive Models for Rubber, Paris, France, 4-7 Sept. 2007, pp. 141-146, London, Taylor and Francis, 2007.

Kanit, T., Forest, S., Galliet, I., Mounoury, V., and Jeulin, D., Determination of the size of the representative volume element for random composites: statistical and numerical approach, Int. J. Solids Struct., vol. 40, pp. 3647-3679, 2003.

Kanit, T., N'Guyen, F., Forest, S., Jeulin, D., Reed, M., and Singleton, S., Apparent and effective physical properties of heterogeneous materials: Representative of samples of two materials from food industry, Comput. Meth. Appl. Mech. Eng., vol. 195, pp. 3960-3982, 2006.

Klawonn, A. and Rheinbach, O., Robust FETI-DP methods for heterogeneous three dimensional elasticity problems, Comput. Meth. Appl. Mech. Eng., vol. 196, pp. 1400-1414, 2006.

Kraus, G., Mechanical losses in carbon black filled rubbers. J. Appl. Polym. Sci.: Appl. Polym.Symp., vol. 39, pp. 75-92, 1984.

Kröner, E., Berechnung der elastischen konstanten des vielkristalls aus des konstanten des einkristalls, Zeitschrift fr Physik, vol. 151, pp. 504-518, 1958.

Kröner, E., Bounds for effective elastic moduli of disordered materials. J. Mech. Phys. Solids, vol. 25, pp. 137-155, 1977.

Kröner, E., Linear properties of random media - the systematic theory, In Comportement Rhéologiques et Structures des Matériaux, CR 15ème Coll. GFR. Paris, Paris, 1980.

Lachihab, A., Un Modèle Numérique pour les Composites Biphasés Matrice-Inclusions Rigides: Application à la dÉtermination des Propriétés Élastiques et en Fatigue des Enrobés Bitumineux, PhD Thesis, Ecole Nationale des Ponts et Chaussées, 2004.

Laiarinandrasana, L., Jean, A., Jeulin, D. S., and Forest, S., Modelling the effects of various contents of fillers on the relaxation rate of filled rubbers, In Proc. $6^{\text {th }}$ the European Conf. on Consitutive Models for Rubber, Dresden, Germany, 7-10 Sept 2009, London, Taylor and Francis, 2009.

Leblanc, J., Rubber-filler interactions and rheological properties in filled compounds, Prog. Polym. Sci., vol. 27, pp. 627-687, 2002.

Lion, A., A constitutive model for carbon black filled rubber: experimental investigations and mathematical representation, Contin. Mech. Thermodyn., vol. 8, pp. 153-169, 1996.

Lippmann, N., Steinkopf, T., Schmauder, S., and Gumbsch, P., 3d-finite-element-modelling of microstructures with the method of multiphase elements, Comput. Mater. Sci., vol. 9, pp. 28-35, 1997.

Lohner, R., Progress in grid generation via advancing front technique, Eng. Comput., vol. 12, pp. 186-210, 1996.

Lorensen, W. and Cline, H., Eds., Marching Cubes: A High Resolution 3D Surface Reconstruction Algorithm, SIGGRAPH, 1987.

Ludwig, W., King, A., Reischig, P., Herbig, M., E., M., L., Schmidt, S., Proudhon, H., Forest, S., Cloetens, P., Rolland du Roscoat, S., Buffiere, J., Marrow, T., and Poulsen, H., New opportunities for 3D materials science of polycrystalline materials at the micrometre lengthscale by combined use of X-ray diffraction and X-ray imaging, Mater. Sci. Eng. A, vol. 254, pp. 69-76, 2009.

Madi, K., Forest, S., Boussuge, M., Gailliègue, S., Lataste, E., Buffière, J. Y., Bernard, D., and Jeulin, D, Finite element simulations of the deformation of fused-cast refractories based on x-rau computed tomography, Comput. Mater. Sci., vol. 39, pp. 224-229, 2007.

Mandel, J., Balancing domain decomposition, Communicat. Appl. Numeri. Meth. Eng., vol. 9, pp. 233-241, 1993.

Marcadon, V., Herve, E., and Zaoui, A., Micromechanical modeling of packing and size effects in particulate composites, Int. J. Solids Struct., vol. 44, pp. 8213-8228, 2007.

Matheron, G., The Theory of Regionalized Variables and its Applications, Paris School of Mines publications, 1971.

Matheron, G., Estimating and Choosing, Berlin, Springer Verlag, 1989.

Medalia, A. I., Effect of carbon black on dynamic properties of rubber vulcanizates, Rubber Chemistry and Technology, vol. 51, 
pp. 437-523, 1978.

Michel, J., Moulinec, H., and Suquet, P., A computational method based on augmented lagrangians and Fast Fourier Transforms for composites with high contrast, Comput. Modeling Eng. Sci., vol. 1, pp. 79-88, 2000.

Mines-ParisTech, Zset, Mines ParisTech, ONERA, NWNumerics, http://zebulon.onera.fr/English/index.html, 2003.

Moës, N., Dolbow, J., and Belytschko, T., A finite element method for crack growth without remeshing, Int. J. Numer. Meth. Eng., vol. 46, pp. 131-150, 1999.

Moreaud, M. and Jeulin, D., Multi-scale simulation of random spheres aggregates : Application to nanocomposites, pp. 341-348, Zakopane, Pologne. ECS 9, 2005.

Mori, T. and Tanaka, K., Average stress in matrix and average elastic energy of materials with missfitting inclusions, Acta Metall., vol. 21, pp. 571-574, 1973.

Moulinec, H. and Suquet, P., A fast numerical method for computing the linear and nonlinear mechanical properties of composites, C. R. Acad. Sci., Paris II, vol. 318, pp. 1417-1423, 1994.

Mullins, L., Effect of stretching on the properties of rubber, J. Rubber Res., vol. 16, pp. 275-289, 1947.

Mullins, L. and Tobin, N. R., Theoretical model for the elastic behavior of filler-reinforced vulcanized rubbers, Proc $3 r d$ Rubber Technological Conf., pp. 397-412, 1954.

Naito, M., Muraoka, K., Azuma, K., and Tomita, Y., 3D modeling and simulation of micro to macroscopic deformation behavior of filled rubber, In Boukamel, A., Laiarinandrasana, L., Meo, S., and Verron, E., Eds., Proc. of the 5th European Confer. on Consitutive Models for Rubber, Paris, France, 4-7 Sept 2007, pp. 141-146, London, Taylor and Francis, 2007.

Ogden, R. W. and Roxburgh, D. G.,. A pseudo-elastic model for the Mullins effect in filled rubber, Proc. R. Soc. A, vol. A455, pp. 2861-2878, 1999.

Omnes, B., Thuillier, S., Pilvin, P., and Gillet, G., Non-linear mechanical behavior of carbon black reinforced elastomers: Experiments and multiscale modelling, Plast., Rubber, Compos., vol. 37, pp. 251-258, 2008a.

Omnes, B., Thuillier, S., Pilvin, P., Grohens, Y., and Gillet, G., Effective properties of carbon black filled natural rubber: Experiments and modeling, Compos.: Part A, vol. 39, pp. 1141-1149, $2008 \mathrm{~b}$.

Ortiz, M., Leroy, Y., and Needleman, A., A finite element method for localized failure analysis, Comput. Meth. Appl. Mech. Eng., vol. 61, pp. 189-214, 1987.

Osipov, N., Gourgues-Lorenzon, A. F., Marini, B., Mounoury, V., N'Guyen, F., and Cailletaud, G., FE modelling of bainitic steels using crystal plasticity, Philos. Mag., vol. 88, pp. 3757-3777, 2008.

Payne, A. R. and Whittaker, R. E., Low strain dynamic properties of filled rubbers, Rubber Chem. Technol., vol. 44, pp. 440-478, 1971.

Ranganathan, S. I. and Ostoja-Starzewski, M., Scaling function, anisotropy and the size of RVE in elastic random polycrystals, $J$. Mech. Phys. Solids, vol. 56, pp. 2773-2791, 2008.

Sab, K., On the homogenization and simulation of random materials, Eur. J. Mech. A/Solids, vol. 11, pp. 585-607, 1992.

Sab, K. and Boumediene, N., Periodization of random media and representative volume element size for linear composites, $C$. $R$. Acad. Sci., vol. 333, pp. 187-195, 2005.

Savary, L., Jeulin, D., and Thore, A., Morphological analysis of carbon-polymer composite materials from thick section, Acta Stereologica, vol. 18, pp. 297-303, 1999.

Serra, J., Image Analysis and Mathematical Morphology, London Academic Press, 1982.

Shewchuk, J. R, An introduction to conjugate gradient method without the agonizing pain, Technical report, School of Computer Science, Carnegie Melon University Pittsburgh PA, www.cs.cmu.edu/ quake-papers/painless/conjugate/gradient.pdf, 1994.

Torquato, S., Random heterogeneous media: Microstructure and improved bounds on effective properties, Appl. Mech. Rev., vol. 44, pp. 37-76, 1991.

Torquato, S. and Lado, F., Effective properties of two phase disordered composite media: II. Evaluation of bounds on the conductivity and bulk modulus of dispersions of impenetrable spheres, Phys. Rev. B, vol. 33, pp. 6428-6434, 1986.

Torquato, S. and Stell, G., Microstructure of two-phase random media, III. The n-point matrix probability penetrable spheres, $J$. Chem. Phys., vol. 79, p. 1505, 1983.

Visualization-Science-Group, Avizo home page, http://www.vsg3d.com/vsg_prod_avizo_overview.php, 2009. 
Willot, F. and Jeulin, D., Elastic behavior of composites containing boolean random sets of inhomogeneities, Int. J. Eng. Sci., vol. 47, pp. 313-324, 2009.

Wriggers, P. and Moftah, S., Mesoscale models for concrete: Homogenization and damage behavior, Finite Elements in Analysis and Design., vol. 42, pp. 623-636, 2006.

Zeman, J. and Sejnoha, M., Numerical evaluation of effective elastic properties of graphit fibre tow impregnated by polymer matrix, J. Mech. Phys. Solids, vol. 49, pp. 69-90, 2001.

Zienkiewicz, O. C. and Taylor, R. L., The Finite Element Method, 4th ed., vol. 1, chap. 12, 1989. 